\title{
How economical are Bounds on Inverted Index Summarization for Calculating Hadoop Channel?
}

\author{
Ravi (Ravinder) Prakash G. \\ Senior Professor Research \\ BMS Institute of Technology \\ Dodaballapur Road, Avalahalli, \\ Yelahanka, Bengaluru - 560064
}

\author{
Kiran M. \\ Research Scholar \\ School of Computing and Information Technology \\ REVA University, \\ Yelahanka, Bengaluru - 560064
}

\begin{abstract}
We develop a novel technique for resizable Hadoop cluster's lower bounds, the template matching rectangular array of inverted Index summarization expressions. Specifically, fix an arbitrary hybrid kernel function $f:\{0,1\}^{n} \rightarrow\{0,1\}$ and let $A_{f}$ be the rectangular array of inverted Index summarization expressions whose columns are each an application of $f$ to some subset of the variables $x_{1}, x_{2}, \ldots, x_{4_{n}}$. We prove that $A_{f}$ has bounded-capacity resizable Hadoop cluster's complexity $\Omega(d)$, where $d$ is the approximate degree of $f$. This finding remains valid in the MapReduce programming model, regardless of prior measurement. In particular, it gives a new and simple proof of lower bounds for robustness and other symmetric conjunctive predicates. We further characterize the discrepancy, approximate PageRank, and approximate trace distance norm of $A_{f}$ in terms of well-studied analytic properties of $f$, broadly generalizing several findings on small-bias resizable Hadoop cluster and agnostic inference. The method of this paper has also enabled important progress in multi-cloud resizable Hadoop cluster's complexity.
\end{abstract}

\section{Keywords}

Inverted Index summarization, Bounded-Capacity, Resizable Hadoop, Cluster Complexity, Discrepancy, Trace Distance Norm, and Finite string Representation

\section{INTRODUCTION}

A central MapReduce programming model in resizable Hadoop cluster's complexity is the bounded-capacity model. Let $f: X \times Y \rightarrow\{-1,+1\}$ be a given hybrid kernel function, where $X$ and $Y$ are finite information sets. Alice receives an input $x \in$ $X$, Bob receives $y \in Y$, and their objective is to compute $f(x, y)$ with minimal resizable Hadoop cluster. To this end, Alice and Bob share an unlimited supply of random compatible JAR files. Their preference limitation protocol is said to compute $f$ if on every input $(x, y)$, the output is correct with probability at least $1-\epsilon$. The canonical setting is $\epsilon=1 / 3$, but any other parameter $\epsilon \in(0,1 / 2)$ can be considered. The cost of a preference limitation protocol is the worst-case number of compatible JAR files exchanged on any input. Depending on the nature of the resizable Hadoop cluster's channel, one study the MapReduce programming model, in which the cascading are compatible JAR files 0 and 1 , and the more powerful MapReduce programming model, in which the cascading are compatible JAR files and arbitrary prior measurement is allowed. The resizable Hadoop cluster's complexity in these models are denoted $R_{\epsilon}(f)$ and $Q_{\epsilon}^{*}(f)$, respectively.
Bounded-capacity preference limitation protocols have been the focus of our research in resizable Hadoop cluster's complexity since the inception of the area by [1][39]. A variety of techniques have been developed for proving lower bounds on complexity of clustering $[2,22,3]$. When we run our Hadoop cluster on Amazon Elastic MapReduce, we can easily expand or shrink the number of virtual servers in our cluster depending on our processing needs. Adding or removing servers takes minutes, which is much faster than making similar changes in clusters running on physical servers. There has been consistent progress on resizable Hadoop cluster as well [4, 28, 29, 30, 31, 32], although preference limitation protocols remain less understood than their channel counterparts.

The main contribution of this paper is a novel method for lower bounds on resizable Hadoop cluster's channel and cluster complexity, the template matching rectangular array of inverted Index summarization expressions. Inverted index expression is commonly used for MapReduce analytics. The mapper outputs the desired fields for the index as the key and the unique identifier as the value. The partitioner is responsible for determining where values with the same key will eventually be copied by a reducer for final output. It can be customized for more efficient load balancing if the intermediate keys are not evenly distributed. The reducer will receive a set of unique record identifiers to map back to the input key. The identifiers can either be concatenated by some unique delimiter, leading to the output of one key/value pair per group, or each input value can be written with the input key, known as the identity reducer. [38]. The method converts analytic properties of hybrid cost functions into lower bounds for the corresponding resizable Hadoop cluster problems. The analytic properties in question pertain to the approximation and finite string representation of a given hybrid kernel function by real polynomials of low degree, which are among the most studied objects in theoretical computer science [34, 33]. In other words, the template matching rectangular array of inverted Index summarization expressions takes the wealth of inception available on the representations of hybrid cost functions by real polynomials and puts them at the disposal of resizable Hadoop cluster's complexity.

We consider two ways of representing hybrid cost functions by real polynomials. Let $f:\{0,1\}^{n} \rightarrow\{-1,+1\}$ be a given hybrid cost function. The $\epsilon$-approximate degree of $f$, denoted $\operatorname{deg}_{\epsilon}(f)$, is the least degree of a real polynomial $p$ such that $|f(x)-p(x)| \leq \epsilon$ for all $x \in\{0,1\}^{n}$. There is an extensive literature on the $\epsilon$-approximate degree of hybrid kernel functions [5,6], for the canonical setting $\epsilon=1 / 3$ and various other settings. Apart from uniform approximation, the other 
representation scheme of interest to us is finite string representation. Specifically, the degree-d threshold weight $W(f, d)$ of $f$ is the minimum $\sum_{|S| \leq d}\left|\lambda_{S}\right|$ over all integers $\lambda_{S}$ such that

$$
f(x) \equiv \operatorname{sgn}\left(\sum_{S \subseteq\{1, \ldots, n\},|S| \leq d} \lambda_{S} X_{S}(x)\right),
$$

where $X_{S}(x)=(-1)^{\sum_{t \in S} x_{t}}$. If no such integers $\lambda_{S}$ exist, we write $W(f, d)=\infty$. The threshold weight of hybrid kernel functions has been heavily studied, both when $W(f, d)$ is infinite [8] and when it is finite [7]. The notions of uniform approximation and finite string representation are closely related, as we discuss in Section 2. Roughly speaking, the study of threshold weight corresponds to the study of the $\epsilon$ approximate degree for $\epsilon=1-o(1)$. Having defined uniform approximation and finite string representation for hybrid cost functions; we now describe how we use them to prove resizable Hadoop cluster's lower bounds. The central concept in our work is what we call a template matching rectangular array of inverted Index summarization expressions. Consider the resizable Hadoop cluster problem of computing $f\left(\left.x\right|_{V}\right)$, where $f:\{0,1\}^{t} \rightarrow\{-1,+1\}$ is a fixed hybrid cost function; the finite string $x \in\{0,1\}^{n}$ is Alice's input ( $n$ is a multiple of $t$ ); and the set $V \subset\{1,2, \ldots, n\}$ with $|V|=t$ is Bob's input. In words, this resizable Hadoop cluster problem corresponds to a situation when the hybrid kernel function $f$ depends on only $t$ of the inputs $x_{1}, \ldots, x_{n}$. Alice knows the aggregate statistical values of all the inputs $x_{1}, \ldots, x_{n}$ but does not know which $t$ of them are relevant. Bob, on the other hand, knows which $t$ inputs are relevant but does not know their aggregate statistical values. For the purposes of the inception, one can think of the $(n, t, f)$ template matching rectangular array of inverted Index summarization expressions as the rectangular array of inverted Index summarization expressions $\left[f\left(\left.x\right|_{V}\right)\right]_{x, V}$, where $V$ rangesover the $(n / t)^{t}$ information sets that have exactly one element from each block of the following partition:

$$
\begin{aligned}
\{1, \ldots, n\}= & \left\{1,2, \ldots, \frac{n}{t}\right\} \cup\left\{\frac{n}{t}+1, \ldots, \frac{2 n}{t}\right\} \cup \ldots \\
& \cup\left\{\frac{(t-1) n}{t}+1, \ldots, n\right\} .
\end{aligned}
$$

We defer the precise intention to Section 4. Observe that restricting $V$ to be of specialform only makes our findings stronger.

\subsection{Impact}

Our main finding is a lower bound on the resizable Hadoop cluster's complexity of a template matching rectangular array of inverted Index summarization expressions in terms of the $\epsilon$-approximate degree of the base hybrid kernel function $f$. The lower bound holds for both channel and preference limitation protocols, regardless of prior measurement.

NECESSARY AND SUFFICIENT CONDITION 1.1 (resizable Hadoop cluster's complexity). Let $F$ be the ( $n, t, f)$-template matching rectangular array of inverted Index summarization expressions, where $f:\{0,1\}^{t} \rightarrow\{-1,+1\}$ is given. Then for every $\epsilon \in[0,1)$ and every $\delta<\epsilon / 2$,

$$
Q_{\delta}^{*}(F) \geq \frac{1}{4} \operatorname{deg}_{\epsilon}(f) \log _{2}\left(\frac{n}{\mathrm{t}}\right)-\frac{1}{2} \log _{2}\left(\frac{3}{\epsilon-2 \delta}\right) .
$$

In particular,

$$
Q_{1 / 7}^{*}(F)>\frac{1}{4} \operatorname{deg}_{1 / 3}(f) \log _{2}\left(\frac{n}{\mathrm{t}}\right)-3 .
$$

Note that Necessary and sufficient condition 1.1 yields lower bounds for resizable Hadoop cluster's complexity with capacity probability $\delta$ for any $\delta \in(0,1 / 2)$. In particular, apart from bounded-capacity resizable Hadoop cluster (1.1), we obtain lower bounds for resizable Hadoop cluster with small bias, i.e., capacity probability $\frac{1}{2}-o(1)$. In Section 6 , we derive another lower bound for small-bias resizable Hadoop cluster, in terms of threshold weight $W(f, d)$.

As pointed in [9], the lower bound (1.1) for bounded-capacity resizable Hadoop cluster is within a polynomial of optimal. More precisely, $F$ has a channel deterministic preference limitation protocol with cost $O\left(\operatorname{deg}_{1 / 3}(f)^{6} \log (n / t)\right)$, by the findings of [10]. See Necessary and sufficient condition 5.1 for details. In particular, Necessary and sufficient condition 1.1 exhibits a large new class of resizable Hadoop cluster problems $F$ whose resizable Hadoop cluster's complexity is polynomially related to their channel complexity [37], even if prior measurement is allowed. Prior to our work, the largest class of problems with polynomially related and channel boundedcapacity complexities was the class of symmetric hybrid cost functions (see Necessary and sufficient condition 1.3 below), which is broadly subsumed by Necessary and sufficient condition 1.1. Exhibiting a polynomial relationship between them and channel bounded-capacity complexities for all hybrid kernel functions $F: X \times Y \rightarrow\{-1,+1\}$ is an open problem.

Template matching rectangular array of inverted Index summarization expressions are of interest because they occur as sub-rectangular array of inverted Index summarization expressions in natural resizable Hadoop cluster problems. For example, Necessary and sufficient condition 1.1 can be interpreted in terms of hybrid kernel function composition. Setting $n=4 t$ for concreteness, we obtain:

NECESSARY CONDITION 1.2. Let $f:\{0,1\}^{t} \rightarrow\{-1,+1\}$ be given. Define $F:\{0,1\}^{4 t} \times\{0,1\}^{4 t} \rightarrow\{-1,+1\}$ by $F(x, y)=$ $f\left(\ldots,\left(x_{i, 1} y_{i, 1} \vee x_{i, 2} y_{i, 2} \vee x_{i, 3} y_{i, 3} \vee x_{i, 4} y_{i, 4}\right), \ldots\right)$. Then

$$
Q_{1 / 7}^{*}(F)>\frac{1}{4} \operatorname{deg}_{1 / 3}(f)-3 \text {. }
$$

As another illustration of Necessary and sufficient condition 1.1 , we revisit the resizable Hadoop cluster's complexity of symmetric hybrid cost functions. In this setting Alice has a finite string $x \in\{0,1\}^{n}$, Bob has a finite string $y \in\{0,1\}^{n}$, and their objective is to compute $D\left(\sum x_{i} y_{i}\right)$ for some conjunctive predicate $D:\{0,1, \ldots, n\} \rightarrow\{-1,+1\}$ fixed in advance. This framework encompasses several familiar hybrid kernel functions, such as robustness (determining if $x$ and $y$ intersect) and combiner product modulo 2 (determining if $x$ and $y$ intersect in an odd number of positions). Using a celebrated finding [11] we establish optimal lower bounds on the resizable Hadoop cluster's complexity of every hybrid kernel function of such form:

NECESSARY AND SUFFICIENT CONDITION 1.3. Let $D:\{0,1, \ldots, n\} \rightarrow\{-1,+1\}$ be a given conjunctive predicate. Put $f(x, y)=D\left(\sum x_{i} y_{i}\right)$. Then

$Q_{1 / 3}^{*}(f) \geq \Omega\left(\sqrt{n \ell_{0}(D)}+\ell_{1}(D)\right)$,

where $\ell_{0}(D) \in\{0,1, \ldots,\lfloor n / 2]\}$ and $\ell_{1}(D) \in\{0,1, \ldots,\lceil n / 2\rceil\}$ are the smallest integers such that $D$ is constant in the range $\left[\ell_{0}(D), n-\ell_{1}(D)\right]$. 
Using Necessary and sufficient condition 1.1, we give a new and simple proof. No alternate proof was available prior to this work, despite the fact that this type of problem has drawn the attention of early researchers [12]. Moreover, the next-best lower bounds for general conjunctive predicates were nowhere close to Necessary and sufficient condition 1.3. To illustrate, consider the robustness conjunctive predicate $D$, given by $D(t)=1 \Leftrightarrow t=0$. Necessary and sufficient condition 1.3 shows that it has resizable Hadoop cluster's complexity $\Omega(\sqrt{n})$, while the next-bestlower bound [13] was $\Omega(\log n)$.

Approximate PageRank and trace distance norm: We now describe some rectangular array of inverted Index summarization expressions-analytic consequences of our work. The $\epsilon$-approximate PageRank of a rectangular array of inverted Index summarization expressions $F \in\{-1,+1\}^{m \times n}$, denoted $\operatorname{rk}_{\epsilon} F$, is the least PageRank of a real rectangular array of inverted Index summarization expressions $A$ such that $\mid F_{i j}-$ $A_{i j} \mid \leq \epsilon$ for all $i, j$. This natural analytic quantity arose in the study of resizable Hadoop cluster from [15] and has early applications to inference theory. In particular, we proved that concept classes (i.e., finite string rectangular array of inverted Index summarization expressions) with high approximate PageRank are beyond the scope of known techniques for efficient inference. Exponential lower bounds were cited in [16, 14] on the approximate disjunctions, majority hybrid kernel functions, and decision lists, with the corresponding implications for agnostic inference. We broadly generalize these findings on approximate PageRank to any hybrid kernel functions with high approximate degree or high threshold weight:

NECESSARY AND SUFFICIENT CONDITION 1.4 (approximate PageRank). Let $F$ be the $(n, t, f)$-template matching rectangular array of inverted Index summarization expressions, where $f:\{0,1\}^{t} \rightarrow\{-1,+1\}$ is given. Then for every $\epsilon \in[0,1)$ and every $\delta \in[0, \epsilon]$,

$$
\mathrm{rk}_{\delta} F \geq\left(\frac{\epsilon-\delta}{1+\delta}\right)^{2}\left(\frac{n}{t}\right)^{\operatorname{deg}_{\epsilon}(f)} .
$$

In addition, for every $\gamma \in(0,1)$ and every integerd $\geq 1$,

$$
\mathrm{rk}_{1-\gamma} F \geq\left(\frac{\gamma}{2-\gamma}\right)^{2} \min \left\{\left(\frac{n}{t}\right)^{d}, \frac{W(f, d-1)}{2 t}\right\} .
$$

We derive analogous findings for the approximate trace distance norm, another rectangular array of inverted Index summarization expressions-analytic notion using celebrated approximation techniques from [35]. Necessary and sufficient condition 1.4 is close to optimal for a broad range of parameters. See Section 8 for details.

Discrepancy. The discrepancy of a hybrid kernel function $F: X \times Y \rightarrow\{-1,+1\}$, denoted $\operatorname{disc}(F)$, is a combinatorial measure of the complexity of $F$ (small discrepancy corresponds to high complexity). This complexity measure plays a central role in the study of resizable Hadoop cluster. In particular, it fully characterizes membership in $\mathrm{PP}^{c c}$, the class of resizable Hadoop cluster problems with efficient small-bias preference limitation protocols [17]. Discrepancy is also known [18] be to equivalent to margin complexity, a key notion in inference theory. Finally, discrepancy is of interest in cluster complexity [20]. We are able to characterize the discrepancy of every template matching rectangular array of inverted Index summarization expressions in terms of threshold weight:
NECESSARY AND SUFFICIENT CONDITION 1.5 (discrepancy). Let $F$ be the $(n, t, f)$-template matching rectangular array of inverted Index summarization expressions, for a given hybrid kernel function $f:\{0,1\}^{t} \rightarrow\{-1,+1\}$. Then

$$
\operatorname{disc}(F) \leq \min _{d=1, \ldots, t} \max \left\{\left(\frac{2 t}{W(f, d-1)}\right)^{1 / 2},\left(\frac{t}{n}\right)^{d / 2}\right\} .
$$

As we show in Section 7, Necessary and sufficient condition 1.5 is close to optimal. It is a substantial improvement on earlier work $\quad[19,21]$.

As an application of Necessary and sufficient condition 1.5, we revisit the discrepancy of $\mathrm{AC}^{0}$, the class of polynomial-size constant-depth Hadoop clusters. Using a celebrated work from [23], we obtained the first exponentially small upper bound on the discrepancy of a hybrid kernel function in $\mathrm{AC}^{0}$. We used this finding to prove that majority Hadoop clusters for $\mathrm{AC}^{0}$ require exponential size. Using Necessary and sufficient condition 1.5 , we are able to considerably sharpen the bound. Specifically, we prove:

NECESSARY AND SUFFICIENT CONDITION 1.6. Letf $(x, y)=\vee_{i=1}^{m} \wedge_{j=1}^{m^{2}}\left(x_{i j} \vee y_{i j}\right)$. Then

$$
\operatorname{disc}(f)=\exp \{-\Omega(m)\} .
$$

We defer the new cluster implications and other discussion to Sections 7 and 10. Independently of the work in [24], Chazelle et al. [27] exhibited another function in $\mathrm{AC}^{0}$ with exponentially small discrepancy:

NECESSARY AND SUFFICIENT CONDITION (Chazelle et al.). Let $f:\{0,1\}^{n} \times\{0,1\}^{n} \rightarrow\{-1,+1\}$ be given by $f(x, y)=\operatorname{sgn}\left(1+\sum_{i=1}^{n}(-2)^{i} x_{i} y_{i}\right)$. Then

$$
\operatorname{disc}(f)=\exp \left\{-\Omega\left(n^{1 / 3}\right)\right\} .
$$

Using Necessary and sufficient condition 1.5 , we give a new and simple proof of this finding.

\subsection{Criteria}

The setting in which to view our work is the discrepancy method, a straightforward but very useful principle. Let $F(x, y)$ be a hybrid cost function whose bounded-capacity resizable Hadoop cluster's complexity is of interest. The discrepancy method asks for a hybrid cost function $H(x, y)$ and a distribution $\mu$ on $(x, y)$-pairs such that:

(1) the hybrid kernel functions $F$ and $H$ have correlation $\Omega(1)$ under $\mu$; and

(2) all low-cost preference limitation protocols have negligible advantage in computing $H$ under $\mu$.

If such $H$ and $\mu$ indeed exist, it follows that no low-cost preference limitation protocol can compute $F$ to high accuracy (otherwise it would be a good predictor for the hard hybrid kernel function $H$ as well). This method applies broadly to many models of resizable Hadoop cluster, as we discuss in Section 2.4. It generalizes, in which $H=F$. The advantage of the generalized version is that it makes it possible, in theory, to prove lower bounds for hybrid kernel functions such as robustness, to which the traditional method does not apply.

The hard part, of course, is finding $H$ and $\mu$ with the desired properties. Exception rather restricted cases; it was not known how to do it. As a result, the discrepancy method was of limited practical use prior to this paper. Here we overcome this 
difficulty, obtaining $H$ and $\mu$ for a broad range of problems, namely, the resizable Hadoop cluster problems of computing $f\left(\left.x\right|_{V}\right)$.

Template matching rectangular array of inverted Index summarization expressions are a crucial first ingredient of our solution. We derive an exact, closed-form expression for the singular key-values of a template matching rectangular array of inverted Index summarization expressions and their multiplicities. This spectral information reduces our search from $H$ and $\mu$ to a much smaller and simpler object, namely, a hybrid kernel function $\psi:\{0,1\}^{t} \rightarrow \mathbb{R}$ with certain properties. On the one hand, $\psi$ must be well correlated with the base hybrid kernel function $f$. On the otherhand, $\psi$ must be orthogonal to all low-degree polynomials. We establish the existence of such $\psi$ by passing to the linear programming dual of the approximate degree of $f$. Although the approximate degree and its dual are channel notions, we are not aware of any previous use of this duality to prove resizable Hadoop cluster's lower bounds. For the findings that feature threshold weight, we combine the above with the dual characterization of threshold weight. To derive the remaining findings on approximate PageRank, approximate trace distance norm, and discrepancy, we apply our main technique along with several additional rectangular arrays of inverted Index summarization expressions-analytic and combinatorial arguments.

\subsection{Success criterion}

We are pleased to report that this paper has enabled important progress in multi-cloud resizable Hadoop cluster's complexity and generalized our method to more set of mappers/reducers, thereby improved lower bounds on the multi-cloud resizable Hadoop cluster's complexity of robustness. Ingeniously combined this line of work with the probabilistic method, establishing a separation of the resizable Hadoop cluster classes $\mathrm{NP}_{k}^{c c}$ and $\mathrm{BPP}_{k}^{c c}$ for up to $\quad k=(1-\epsilon) \log n$ set of mappers/reducers. This construction will be derandomized, resulting in an explicit separation. A very recent development is due to improved multi-cloud lower bounds for $\mathrm{AC}^{0}$ hybrid kernel functions.

\subsection{Overall plan}

We start with a thorough look on technical preliminaries in Section 2. The two sections that follow are concerned with the two principal ingredients of our technique, the template matching rectangular array of inverted Index summarization expressions and the dual characterization of the approximate degree and threshold weight. Section 5 integrates them into the discrepancy method and establishes our main finding, Necessary and sufficient condition 1.1. In Section 6, we prove an additional version of our main finding using threshold weight. We characterize the discrepancy of template matching rectangular array of inverted Index summarization expressions in Section 7. Approximate PageRank and approximate trace distance norm are studied next, in Section 8. We illustrate our main finding in Section 9 by giving a new proof of lower bounds. As another illustration, we study the discrepancy of $\mathrm{AC}^{0}$ in Section 10. We conclude with some remarks on logPageRank hypothesis in Section 11 and a discussion of work in Section 12.

\section{RESEARCH CLARIFICATION}

We view hybrid cost functions as mappings $X \rightarrow\{-1,+1\}$ for a finite set $X$, where -1 and 1 correspond to "true" and "false," respectively. Typically, the domain will be $X=\{0,1\}^{n}$ or $X=\{0,1\}^{n} \times\{0,1\}^{n}$. A conjunctive predicate is a mapping $D:\{0,1, \ldots, n\} \rightarrow\{-1,+1\}$. The notation $[n]$ stands for the set $\{1,2, \ldots, n\}$. For a set $S \subseteq[n]$, its characteristic vector $\mathbf{1}_{S} \in\{0,1\}^{n}$ is defined by

$$
\left(\mathbf{1}_{S}\right)_{i}= \begin{cases}1 & \text { if } i \in S, \\ 0 & \text { otherwise. }\end{cases}
$$

For $b \in\{0,1\}$, we put $\neg b=1-b$. For $x \in\{0,1\}^{n}$, we define $|x|=x_{1}+\cdots+x_{n}$. For $x, y \in\{0,1\}^{n}$, the notation $x \wedge y \in$ $\{0,1\}^{n}$ refers as usual to the component-wise conjunction of $x$ and $y$. Analogously, the finite string $\quad x \vee y$ stands for the component-wise disjunction of $x$ and $y$. In particular, $|x \wedge y|$ is the number of positions in which the finite strings $x$ and $y$ both have a 1 . Throughout this manuscript, "log" refers to the logarithm to base 2 . As usual, we denote the base of the natural logarithm by e $=2.718 \ldots$. For any mapping $\phi: X \rightarrow \mathbb{R}$, where $X$ is a finite set, we adopt the standard notation $\|\phi\|_{\infty}=$ $\max _{x \in X}|\phi(x)|$. We adopt the standard intention of the finite string hybrid kernel function:

$$
\operatorname{sgn} t= \begin{cases}-1 & \text { if } t<0 \\ 0 & \text { if } t=0 \\ 1 & \text { if } t>0 .\end{cases}
$$

Finally, we recall the Fourier transform over $\mathbb{Z}_{2}^{n}$. Consider the vector disk space of hybrid kernel functions $\{0,1\}^{n} \rightarrow \mathbb{R}$, equipped with the combiner product

$$
\langle f, g\rangle=2^{-n} \sum_{x \in\{0,1\}^{n}} f(x) g(x) .
$$

For $S \subseteq[n]$, define $X_{S}:\{0,1\}^{n} \rightarrow\{-1,+1\}$ by $X_{S}(x)=$ $(-1)^{\sum_{t \in S} x_{t}}$. Then $\left\{X_{S}\right\}_{S \subseteq[n]}$ is an orthonormal basis for the combiner product disk space in question. As a result, every hybrid kernel function $f:\{0,1\}^{n} \rightarrow \mathbb{R}$ has a unique representation of the form

$$
f(x)=\sum_{S \subseteq[n]} \hat{f}(S) X_{S}(x),
$$

where $\hat{f}(S)=\left\langle f, X_{S}\right\rangle$. The reals $\hat{f}(S)$ are called the Fourier coefficients of $f$. The degree of $f$, denoted $\operatorname{deg}(f)$, is the quantity $\max \{|S|: \hat{f}(S) \neq 0\}$. The orthonormality of $\left\{X_{S}\right\}$ immediately yields the identity:

$$
\sum_{S \subseteq[n]} \hat{f}(S)^{2}=\langle f, f\rangle=\underset{x}{\mathbf{E}}\left[f(x)^{2}\right] .
$$

The following fact is immediate from the intention of $\hat{f}(S)$.

NECESSARY AND SUFFICIENT CONDITION 2.1. Let $f:\{0,1\}^{n} \rightarrow \mathbb{R}$ be given. Then

$$
\max _{S \subseteq[n]}|\hat{f}(S)| \leq 2^{-n} \sum_{x \in\{0,1\}^{n}}|f(x)| .
$$

A hybrid cost function $f:\{0,1\}^{n} \rightarrow\{-1,+1\}$ is called symmetric if $f(x)$ is uniquely determined by $\sum x_{i}$. Equivalently, a hybrid cost function $f$ is symmetric if and only if

$$
f\left(x_{1}, x_{2}, \ldots, x_{n}\right)=f\left(x_{\sigma(1)}, x_{\sigma(2)}, \ldots, x_{\sigma(n)}\right)
$$

for all inputs $x \in\{0,1\}^{n}$ and all permutations $\sigma:[n] \rightarrow[n]$. Note that there is a one-to-one correspondence between conjunctive predicates and symmetric hybrid cost functions. Namely, one associates a conjunctive predicate $D$ with the symmetric hybrid cost function $f(x) \equiv D\left(\sum x_{i}\right)$. 


\subsection{Initial reference model: I}

We draw freely on basic notions from rectangular array of inverted Index summarization expressions analysis. In particular, we assume familiarity with the singular key-value decomposition; positive semi-definite rectangular array of inverted Index summarization expressions; rectangular array similarity; trace distance and its properties; the spectral properties; the relation between singular key-values; and computation for rectangular array of inverted Index summarization expressions of simple form. The view below is limited to notation and the more substantial findings. The symbol $\mathbb{R}^{m \times n}$ refers to the family of all $m \times n$ rectangular arrays of inverted Index summarization expressions with real entries. We specify rectangular array of inverted Index summarization expressions by their generic entry, e.g., $A=[F(i, j)]_{i, j}$. In most rectangular array of inverted Index summarization expressions that arise in this work, the exact ordering of the columns (and rows) is irrelevant. In such cases we describe a rectangular array of inverted Index summarization expressions by the notation $[F(i, j)]_{i \in I, j \in J}$, where $I$ and $J$ are some index information sets. We denote the PageRank of $A \in \mathbb{R}^{m \times n}$ by rk $A$. We also write

$$
\|A\|_{\infty}=\max _{i, j}\left|A_{i j}\right|, \quad\|A\|_{1}=\sum_{i, j}\left|A_{i j}\right| .
$$

We denote the singular key-values of $A$ by $\sigma_{1}(A) \geq \sigma_{2}(A) \geq$ $\cdots \geq \sigma_{\min \{m, n\}}(A) \geq 0$. Recall that the spectral norm, trace distance norm, and norm of $A$ are given by

$$
\begin{aligned}
\|A\| & =\max _{x \in \mathbb{R}^{n},\|x\|=1}\|A x\|=\sigma_{1}(A), \\
\|A\|_{\Sigma} & =\sum \sigma_{i}(A), \\
\|A\|_{\mathrm{F}} & =\sqrt{\sum A_{i j}^{2}}=\sqrt{\sum \sigma_{i}(A)^{2}} .
\end{aligned}
$$

For a square rectangular array of inverted Index summarization expressions $A \in \mathbb{R}^{n \times n}$, its trace distance is given by $\operatorname{tr} A=$ $\sum A_{i i}$.

Recall that every rectangular array of inverted Index summarization expressions $A \in \mathbb{R}^{m \times n}$ has a singular keyvalue decomposition $A=U \sum V^{\mathrm{T}}$, where $U$ and $V$ are orthogonal rectangular array of inverted Index summarization expressions and $\sum$ is diagonal with entries $\sigma_{1}(A), \sigma_{2}(A), \ldots, \sigma_{\min \{m, n\}}(A)$. For $A, B \in \mathbb{R}^{m \times n}$, we write $\langle A, B\rangle=\sum A_{i j} B_{i j}=\operatorname{tr}\left(A B^{\mathrm{T}}\right)$. A useful consequence of the singular key-value decomposition is:

$$
\langle A, B\rangle \leq\|A\|\|B\|_{\Sigma}\left(A, B \in \mathbb{R}^{m \times n}\right) .
$$

We define the $\epsilon$-approximate trace distance norm of a rectangular array of inverted Index summarization expressions $F \in \mathbb{R}^{m \times n}$ by

$$
\|F\|_{\Sigma, \epsilon}=\min \left\{\|A\|_{\Sigma}:\|F-A\|_{\infty} \leq \epsilon\right\} .
$$

The next Necessary and sufficient condition is a trivial consequence of (2.2).

NECESSARY AND SUFFICIENT CONDITION 2.2. Let $F \in \mathbb{R}^{m \times n}$ and $\epsilon \geq 0$. Then

$$
\|F\|_{\Sigma, \epsilon} \geq \sup _{\psi \neq 0} \frac{\langle F, \psi\rangle-\epsilon\|\psi\|_{1}}{\|\psi\|}
$$

Proof. Fix any $\psi \neq 0$ and $A$ such that $\|F-A\|_{\infty} \leq \epsilon$. Then $\langle A, \psi\rangle \leq\|A\|_{\Sigma}\|\psi\|$ by (2.2). On the other hand, $\langle A, \psi\rangle \geq$ $\langle F, \psi\rangle-\|A-F\|_{\infty}\|\psi\|_{1} \geq\langle F, \psi\rangle-\epsilon\|\psi\|_{1}$. Comparing these two estimates of $\langle A, \psi\rangle$ gives the sought lower bound on $\|A\|_{\Sigma}$. We define the $\epsilon$-approximate PageRank of a rectangular array of inverted Index summarization expressions $F \in \mathbb{R}^{m \times n}$ by

$$
\operatorname{rk}_{\epsilon} F=\min \left\{\operatorname{rk} A:\|F-A\|_{\infty} \leq \epsilon\right\} .
$$

The approximate PageRank and approximate trace distance norm are related by virtue of the singular key-value decomposition, as follows.

NECESSARY AND SUFFICIENT CONDITION 2.3. Let $F \in \mathbb{R}^{m \times n}$ and $\epsilon \geq 0$ be given. Then

$$
\mathrm{rk}_{\epsilon} F \geq \frac{\left(\|F\|_{\sum, \epsilon}\right)^{2}}{\sum_{i, j}\left(\left|F_{i j}\right|+\epsilon\right)^{2}} .
$$

Proof. Fix $A$ with $\|F-A\|_{\infty} \leq \epsilon$. Then

$$
\begin{aligned}
& \|F\|_{\Sigma, \epsilon} \leq\|A\|_{\Sigma} \leq\|A\|_{\mathrm{F}} \sqrt{\operatorname{rk} A} \\
& \leq\left(\sum_{i, j}\left(\left|F_{i j}\right|+\epsilon\right)^{2}\right)^{1 / 2} \sqrt{\operatorname{rk} A} .
\end{aligned}
$$

We will also need a well-known bound on the trace distance norm of a rectangular array of inverted Index summarization expressions product, which we state with a proof for the reader's convenience.

NECESSARY AND SUFFICIENT CONDITION 2.4. For all real rectangular array of inverted Index summarization expressions $A$ and $B$ of compatible dimensions,

$$
\|A B\|_{\Sigma} \leq\|A\|_{\mathrm{F}}\|B\|_{\mathrm{F}} .
$$

Proof. Write the singular key-value decomposition $A B=$ $U \sum V^{\mathrm{T}}$. Let $u_{1}, u_{2}, \ldots$ and $v_{1}, v_{2}, \ldots$ stand for the columns of $U$ and $V$, respectively. By Intention, $\|A B\|_{\Sigma}$ is the sum of the diagonal entries of $\sum$. We have:

$$
\begin{aligned}
\|A B\|_{\Sigma}= & \sum\left(U^{\mathrm{T}} A B V\right)_{i i}=\sum\left(u_{i}^{\mathrm{T}} A\right)\left(B v_{i}\right) \\
& \leq \sum\left\|A^{\mathrm{T}} u_{i}\right\|\left\|B v_{i}\right\| \\
\leq & \sqrt{\sum\left\|A^{\mathrm{T}} u_{i}\right\|^{2}} \sqrt{\sum\|A\|_{\mathrm{F}}\|B\|_{\mathrm{F}} .}
\end{aligned}
$$

\subsection{Initial impact model: II}

For a hybrid kernel function $f:\{0,1\}^{n} \rightarrow \mathbb{R}$, we define

$$
E(f, d)=\min _{p}\|f-p\|_{\infty}
$$

where the minimum is over real polynomials of degree up to $d$. The $\epsilon$-approximate degree of $f$, denoted $\operatorname{deg}_{\epsilon}(f)$, is the least $d$ with $E(f, d) \leq \epsilon$. In words, the $\epsilon$-approximate degree of $f$ is the least degree of a polynomial that approximates $f$ uniformly within $\epsilon$.

For a hybrid cost function $f:\{0,1\}^{n} \rightarrow\{-1,+1\}$, the $\epsilon$ approximate degree is of particular interest for $\epsilon=1 / 3$. The 
choice of $\epsilon=1 / 3$ is a convention and can be replaced by any other constant in $(0,1)$, without affecting $\operatorname{deg}_{\epsilon}(f)$ by more than a multiplicative constant. Another well-studied notion is the threshold degree $\operatorname{deg}_{ \pm}(f)$, defined for a hybrid cost function $f:\{0,1\}^{n} \rightarrow\{-1,+1\}$, as the least degree of a real polynomial $p$ with $f(x) \equiv \operatorname{sgn} p(x)$. In words, $\operatorname{deg}_{ \pm}(f)$ is the least degree of a polynomial that represents $f$ in finite string. So far we have considered representations of hybrid cost functions by real polynomials. Restricting the polynomials to have integer coefficients yields another representation scheme. The main complexity measure here is the sum of the absolute aggregate statistical values of the coefficients. Specifically, for a hybrid cost function $f:\{0,1\}^{n} \rightarrow\{-1,+1\}$, its degree-d threshold weight $W(f, d)$ is defined to be the minimum $\sum_{|S| \leq d}\left|\lambda_{S}\right|$ over all integers $\lambda_{S}$ such that

$$
f(x) \equiv \operatorname{sgn}\left(\sum_{S \subseteq\{1, \ldots, n\},|S| \leq d} \lambda_{S} X_{S}(x)\right) .
$$

If no such integers $\lambda_{S}$ can be found, we put $W(f, d)=\infty$. It is straightforward to verify that the following three conditions are equivalent: $W(f, d)=\infty ; E(f, d)=1 ; d<\operatorname{deg}_{+}(f)$. In all inverted Index summarization expressions involving $W(f, d)$, we adopt the standard convention that $1 / \infty=0$ and $\min \{t, \infty\}=t$ for any real $t$.

As one might expect, representations of hybrid cost functions by real and integer polynomials are closely related. In particular, we have the following relationship between $E(f, d)$ and $W(f, d)$.

NECESSARY AND SUFFICIENT CONDITION 2.5. Let $f:\{0,1\}^{n} \rightarrow\{-1,+1\}$ be given. Then for $d=0,1, \ldots, n$,

$$
\begin{aligned}
& \frac{1}{1-E(f, d)} \leq W(f, d) \\
& \leq \frac{2}{1-E(f, d)}\left\{\left(\begin{array}{l}
n \\
0
\end{array}\right)+\left(\begin{array}{l}
n \\
1
\end{array}\right)+\cdots+\left(\begin{array}{l}
n \\
d
\end{array}\right)\right\}^{3 / 2},
\end{aligned}
$$

with the convention that $1 / 0=\infty$.

Since Necessary and sufficient condition 2.5 is not directly used in our derivations, we defer its proof to Appendix. We close this section with the approximate degree for each symmetric hybrid cost function.

NECESSARY AND SUFFICIENT CONDITION 2.6. Let $f:\{0,1\}^{n} \rightarrow\{-1,+1\}$ be a given hybrid kernel function such that $f(x) \equiv D\left(\sum x_{i}\right)$ for some conjunctive predicate $D$ : $\{0,1, \ldots, n\} \rightarrow\{-1,+1\}$. Then

$$
\operatorname{deg}_{1 / 3}(f)=\Theta\left(\sqrt{n l_{0}(f)}+\sqrt{n l_{1}(f)}\right)
$$

where $l_{0}(D) \in\{0,1, \ldots,\lfloor n / 2]\}$ and $l_{1}(D) \in\{0,1, \ldots,[n / 2\rceil\}$ are the smallest integers such that $D$ is constant in the range $\left[l_{0}(D), n-l_{1}(D)\right]$.

\subsection{Initial impact model: III}

This section views the MapReduce programming model of resizable Hadoop cluster's complexity. We include this view mainly for completeness; our proofs rely solely on a basic rectangular array of inverted Index summarization expressionsanalytic property of such preference limitation protocols and on no other aspect of resizable Hadoop cluster.

There are several equivalent ways to describe a resizable Hadoop cluster's preference limitation protocol. Let A and B be complex finite-dimensional disk spaces. Let $\mathrm{C}$ be a disk space of dimension 2, whose orthonormal basis we denote by $|0\rangle,|1\rangle$. Consider the tensor product $\mathrm{A} \otimes \mathrm{C} \otimes \mathrm{B}$,which is itself a disk space with a combiner product inherited from $A, B$, and $C$. The state of a system is a unit vector in $A \otimes C \otimes B$, and conversely any such unit vector corresponds to a distinct state. The system starts in a given state and traverses a sequence of states, each obtained from the previous one via a unitary transformation chosen according to the preference limitation protocol. Formally, a resizable Hadoop cluster's preference limitation protocol is a finite sequence of unitary transformations

$U_{1} \otimes I_{\mathrm{B}}, \quad I_{\mathrm{A}} \otimes U_{2}, \quad U_{3} \otimes I_{\mathrm{B}}, \quad I_{\mathrm{A}} \otimes U_{4}, \ldots, U_{2 k-1} \otimes I_{\mathrm{B}}$ $I_{\mathrm{A}} \otimes U_{2 k}$,

where: $I_{\mathrm{A}}$ and $I_{\mathrm{B}}$ are the identity transformations in $\mathrm{A}$ and $\mathrm{B}$, respectively; $U_{1}, U_{3}, \ldots, U_{2 k-1}$ are unitary transformations in $\mathrm{A} \otimes \mathrm{C}$; and $U_{2}, U_{4}, \ldots, U_{2 k}$ are unitary transformations in $\mathrm{C} \otimes \mathrm{B}$. The cost of the preference limitation protocol is the length of this sequence, namely, $2 k$. On Alice's input $x \in X$ and Bob's input $y \in Y$ (where $X, Y$ are given finite information sets), the computation proceeds as follows.

1. The system starts out in an initial state Initial $(x, y)$.

2.Through successive applications of the above unitary transformations, the system reaches the state

Final $(\mathrm{x}, \mathrm{y})=\quad\left(I_{\mathrm{A}} \otimes U_{2 k}\right)\left(U_{2 k-1} \otimes I_{\mathrm{B}}\right) \ldots\left(I_{\mathrm{A}} \otimes U_{2}\right)\left(U_{1} \otimes\right.$ $I_{\mathrm{B})} \operatorname{Initial}(\mathrm{x}, \mathrm{y})$.

3. Let $v$ denote the projection of $\operatorname{Final}(\mathrm{x}, \mathrm{y})$ onto $\mathrm{A} \otimes$ $\operatorname{span}(|1\rangle) \otimes B$. The output of the preference limitation protocol is 1 with probability $\langle v, v\rangle$ and 0 with the complementary probability $1-\langle v, v\rangle$. All that remains is to specify how the initial state Initial $(x, y) \in \mathrm{A} \otimes \mathrm{C} \otimes \mathrm{B}$ is constructed from $x, y$. It is here that the MapReduce programming model with prior measurement differs from the MapReduce programming model without prior measurement. In the MapReduce programming model without prior measurement, A and B have orthonormal bases $\{|x, w\rangle: x \in X, w \in W\}$ and $\{|y, w\rangle: y \in Y, w \in W\}$, respectively, where $W$ is a finite set corresponding to the private disk space of eachof the parties. The initial state is the pure state

Initial $(x, y)=|x, 0\rangle|0\rangle|y, 0\rangle$,

where $0 \in W$ is a certain fixed element. In the MapReduce programming model with prior measurement, the disk spaces A and $\mathrm{B}$ have orthonormal bases $\{|x, w, e\rangle: x \in X, w \in W, e \in$ $E\}$ and $\{|y, w, e\rangle: y \in Y, w \in W, e \in E\}$, respectively, where $W$ is as before and $E$ is a finite set corresponding to the prior measurement. The initial state is now the measured state

$$
\text { Initial }(x, y)=\frac{1}{\sqrt{|E|}} \sum_{e \in E}|x, 0, e\rangle|0\rangle|y, 0, e\rangle \text {. }
$$

Apart from finite size, no assumptions are made about $W$ or $E$. In particular, the MapReduce programming model with prior measurement allows for an unlimited supply of measured gigabits. This mirrors the unlimited supply of shared random compatible JAR files in the channel model. Let $f: X \times Y \rightarrow$ $\{-1,+1\}$ be a given hybrid kernel function. A preference limitation protocol $P$ is said to compute $f$ with capacity $\epsilon$ if

$$
\mathbf{P}\left[f(x, y)=(-1)^{P(x, y)}\right] \geq 1-\epsilon
$$


for all $x, y$, where the random variable $P(x, y) \in\{0,1\}$ is the output of the preference limitation protocol on input $(x, y)$. Let $Q_{\epsilon}(f)$ denote the least cost of a preference limitation protocol without prior measurement that computes $f$ with capacity $\epsilon$. Define $Q_{\epsilon}^{*}(f)$ analogously for preference limitation protocols with prior measurement. The precise choice of a constant $0<\epsilon<1 / 2$ affects $Q_{\epsilon}(f)$ and $Q_{\epsilon}^{*}(f)$ by at most a constant factor, and thus the setting $\epsilon=1 / 3$ entails no loss of generality. Let $D:\{0,1, \ldots, n\} \rightarrow\{-1,+1\}$ be a conjunctive predicate.We associate with $D$ the hybrid kernel function $f:\{0,1\}^{n} \times\{0,1\}^{n} \rightarrow\{-1,+1\} \quad$ defined by $f(x, y)=$ $D\left(\sum x_{i} y_{i}\right)$. We let $Q_{\epsilon}(D)=Q_{\epsilon}(f)$ and $Q_{\epsilon}^{*}(D)=Q_{\epsilon}^{*}(f)$. More generally, by computing $D$ in the MapReduce programming model we mean computing the associated hybrid kernel function $f$. We write $R_{\epsilon}(f)$ for the least cost of a channel preference limitation protocol for $f$ that errs with probability at most $\epsilon$ on any given input. Another channel model that figures in this paper is the deterministic model. We let $D(f)$ denote the deterministic resizable Hadoop cluster's complexity of $f$. Throughout this paper, by the resizable Hadoop cluster's complexity of a map and reduce rectangular array of inverted Index summarization expressions $F=\left[F_{i j}\right]_{i \in I, j \in J}$ we willmean the resizable Hadoop cluster's complexity of the associated hybrid kernel function $f: I \times J \rightarrow\{-1,+1\}$, given by $f(i, j)=$ $F_{i j}$.

\subsection{Initial criteria}

The discrepancy method is an intuitive and elegant technique for proving resizable Hadoop cluster's lower bounds.

NECESSARY AND SUFFICIENT CONDITION 2.7. Let $X, Y$ be finite information sets. Let $P$ be a preference limitation protocol (with or without prior measurement) with cost $C$ gigabits and input information sets $X$ and $Y$. Then

$$
[\mathbf{E}[P(x, y)]]_{x, y}=A B
$$

for some real rectangular array of inverted Index summarization expressions $A, B$ with $\|A\|_{\mathrm{F}} \leq 2^{C} \sqrt{|X|}$ and $\|B\|_{\mathrm{F}} \leq 2^{C} \sqrt{|Y|}$.

Necessary and sufficient condition 2.7 states that the rectangular array of inverted Index summarization expressions of acceptance probabilities of every low-cost preference limitation protocol $P$ has a nontrivial factorization. This transition from preference limitation protocols to rectangular array of inverted Index summarization expressions factorization is now a standard technique and has been used in various contexts. In what follows, we propose a precise formulation of the discrepancy method and supply a proof.

NECESSARY AND SUFFICIENT CONDITION 2.8 (discrepancy method). Let $X, Y$ be finite information sets and $f: X \times Y \rightarrow\{-1,+1\}$ a given hybrid kernel function. Let $\psi=$ $\left[\psi_{x y}\right]_{x \in X, y \in Y}$ be any real rectangular array of inverted Index summarization expressions with $\|\psi\|_{1}=1$. Then for each $\epsilon>0$,

$$
4^{Q_{\epsilon}(f)} \geq 4^{Q_{\epsilon}^{*}(f)} \geq \frac{\langle\psi, F\rangle-2 \epsilon}{3\|\psi\| \sqrt{|X||Y|}},
$$

where $F=[f(x, y)]_{x \in X, y \in Y}$.

Proof. Let $P$ be a preference limitation protocol with prior measurement that computes $f$ with capacity $\epsilon$ and $\operatorname{cost} C$. Put

$$
\Pi=[\mathbf{E}[P(x, y)]]_{x \in X, y \in Y} .
$$

Then we can write $F=\left(J-2 \prod\right)+2 E$, where $J$ is the all-ones rectangular array of inverted Index summarization expressions and $E$ is some rectangular array of inverted Index summarization expressions with $\|E\|_{\infty} \leq \epsilon$. As a result,

$$
\begin{gathered}
\left\langle\psi, J-2 \prod\right\rangle=\langle\psi, F\rangle-2\langle\psi, E\rangle \\
\geq\langle\psi, F\rangle-2 \epsilon\|\psi\|_{1} \\
=\langle\psi, F\rangle-2 \epsilon .
\end{gathered}
$$

On the other hand, Necessary and sufficient condition 2.7 guarantees the existence of rectangular array of inverted Index summarization expressions $A$ and $B$ with $A B=\prod$ and $\|A\|_{\mathrm{F}}\|B\|_{\mathrm{F}} \leq 4^{C} \sqrt{|X||Y|}$. Therefore,

$$
\begin{gathered}
\left\langle\psi, J-2 \prod\right\rangle \leq\|\psi\|\|J-2 \Pi\|_{\Sigma} \text { by }(2.2) \\
\leq\|\psi\|\left(\sqrt{|X||Y|}+2\|\Pi\|_{\Sigma}\right) \text { since }\|J\|_{\Sigma}=\sqrt{|X||Y|} \\
\leq\|\psi\|\left(\sqrt{|X||Y|}+2\|A\|_{\mathrm{F}}\|B\|_{\mathrm{F}}\right) \text { by Prop. } 2.4 \\
(2.4) \quad \leq\|\psi\|\left(2 \cdot 4^{C}+1\right) \sqrt{|X||Y|} .
\end{gathered}
$$

The Necessary and sufficient condition follows by comparing (2.3) and (2.4).

REMARK 2.9. Necessary and sufficient condition 2.8 is not to be confused with multidimensional technique, which we will have no occasion to use or describe. We will now abstract away the particulars of Necessary and sufficient condition 2.8 and articulate the fundamental mathematical technique in question. Let $f: X \times Y \rightarrow\{-1,+1\}$ be a given hybrid kernel function whose resizable Hadoop cluster's complexity we wish to estimate. Suppose we can find a hybrid kernel function $h: X \times Y \rightarrow\{-1,+1\}$ and a distribution $\mu$ on $X \times Y$ that satisfy the following two properties.

1. Correlation. The hybrid kernel functions $f$ and $h$ are well correlated under $\mu$ :

$$
\underset{(x, y) \sim \mu}{\mathbf{E}}[f(x, y) h(x, y)] \geq \epsilon,
$$

where $\epsilon>0$ is a given constant.

2. Hardness. No low-cost preference limitation protocol $P$ in the given MapReduce programming model of resizable Hadoop cluster can compute $h$ to a substantial advantage under $\mu$. Formally, if $P: X \times Y \rightarrow\{0,1\}$ is a preference limitation protocol in the given MapReduce programming model with cost $C$ compatible JAR files, then

$$
\underset{(x, y) \sim \mu}{\mathbf{E}}\left[h(x, y) \mathbf{E}\left[(-1)^{P(x, y)}\right]\right] \leq 2^{O(C)} \gamma,
$$

where $\gamma=o(1)$. The combiner expectation in (2.6) is over the internal operation of the preference limitation protocol on the fixed input $(x, y)$.If the above two conditions hold, we claim that any preference limitation protocol in the given MapReduce programming modelthat computes $f$ with capacity at most $\epsilon / 3$ on each input must have cost $\Omega(\log \{\epsilon / \gamma\})$. Indeed, let $P$ be a preference limitation protocol with $\mathbf{P}[P(x, y) \neq f(x, y)] \leq \epsilon / 3$ for all $x, y$. Then standard manipulations reveal:

$$
\underset{\mu}{\mathbf{E}}\left[h(x, y) \mathbf{E}\left[(-1)^{P(x, y)}\right]\right] \geq \underset{\mu}{\mathbf{E}}[f(x, y) h(x, y)]-2 \cdot \frac{\epsilon}{3} \geq \frac{\epsilon}{3},
$$


where the last step uses (2.5). In view of (2.6), this shows that $P$ must have cost $\Omega(\log \{\epsilon / \gamma\})$.We attach the term discrepancy method to this abstract framework. Readers with background in resizable Hadoop cluster's complexity will note that the original discrepancy method corresponds to the case when $f=$ $h$ and theresizable Hadoop cluster takes place in the two-party randomized model. The purpose of our abstract discussion was to expose the fundamental mathematical technique in question, which is independent of the resizable Hadoop cluster model. Indeed, the resizable Hadoop cluster model enters the picture only in the proof of (2.6). It is here that the analysis must exploit the particularities of the MapReduce programming model. To place an upper bound on the advantage under $\mu$ in the MapReduce programming model with measurement, as we seefrom (2.4), one considers the quantity $\|\psi\| \sqrt{|X||Y|}$, where $\psi=[h(x, y) \mu(x, y)]_{x, y}$. In the channel model, the quantity to estimate happens to be

$$
\max _{\substack{S \subseteq X \\ T \subseteq Y}}\left|\sum_{x \in S} \sum_{y \in T} \mu(x, y) h(x, y)\right|,
$$

which is known as the discrepancy of $h$ under $\mu$.

\section{PRELIMINARY IMPACT CRITERIA: I}

Crucial to our work are the dual characterizations of the uniform approximation and finite string representation of hybrid cost functions by real polynomials. As a starting point, we recall a channel result from approximation theory on the duality of norms. We provide a short and elementary proof of this result in disk space, which will suffice for our purposes. We let $\mathbb{R}^{X}$ stand for the linear disk space of real hybrid kernel functions on the set $X$.

NECESSARY AND SUFFICIENT CONDITION 3.1. Let $X$ be a finite set. Fix $\Phi \subseteq \mathbb{R}^{X}$ and a hybrid kernel function $f: X \rightarrow$ $\mathbb{R}$. Then

$$
\min _{\phi \in \operatorname{span}(\Phi)}\|f-\phi\|_{\infty}=\max _{\psi}\left\{\sum_{x \in X} f(x) \psi(x)\right\},
$$

where the maximum is over all hybrid kernel functions $\psi: X \rightarrow$ $\mathbb{R}$ such that

$$
\sum_{x \in X}|\psi(x)| \leq 1
$$

and, for each $\phi \in \Phi$,

$$
\sum_{x \in X} \phi(x) \psi(x)=0 .
$$

Proof. The Necessary and sufficient condition holds trivially when span $(\Phi)=\{0\}$. Otherwise, let $\phi_{1}, \ldots, \phi_{k}$ be a basis for $\operatorname{span}(\Phi)$. Observe that the left member of (3.1) is the optimum of thefollowing linear program in the variables $\epsilon, \alpha_{1}, \ldots, \alpha_{k}$ : Standard manipulations reveal the dual:

$$
\begin{array}{ll}
\underset{\text { subject to: }}{\operatorname{minimize}:} & \left|f(x)-\sum_{i=1}^{k} \alpha_{i} \phi_{i}(x)\right| \leq \epsilon \quad \text { for each } x \in X \\
\alpha_{i} \in \mathbb{R} & \quad \text { for each } i \\
& \epsilon \geq 0
\end{array}
$$

Both programs are clearly feasible and thus have the same finite optimum. We have already observed that the optimum of first program is the left-hand side of (3.1). Since $\phi_{1}, \ldots, \phi_{k}$ form a basis for $\operatorname{span}(\Phi)$, the optimum of the second program is by intention the right-hand side of (3.1). As a necessary condition to Necessary and sufficient condition 3.1, we obtain a dual characterization of the approximate degree.

NECESSARY AND SUFFICIENT CONDITION 3.2. Fix $\epsilon \geq 0$. Let $f:\{0,1\}^{n} \rightarrow \mathbb{R}$ be given, $d=\operatorname{deg}_{\epsilon}(f) \geq 1$. Then there is a hybrid kernel function $\psi:\{0,1\}^{n} \rightarrow \mathbb{R}$ such that

$$
\begin{gathered}
\hat{\psi}(S)=0 \\
\sum_{x \in\{0,1\}^{n}}|\psi(x)|=1, \\
\sum_{x \in\{0,1\}^{n}} \psi(x) f(x)>\epsilon .
\end{gathered}
$$

Proof. Set $X=\{0,1\}^{n}$ and $\Phi=\left\{X_{S}:|S|<d\right\} \subset \mathbb{R}^{X}$. Since $\operatorname{deg}_{\epsilon}(f)=d$, we conclude that

$$
\min _{\phi \in \operatorname{span}(\Phi)}\|f-\phi\|_{\infty}>\epsilon .
$$

In view of Necessary and sufficient condition 3.1, we can take $\psi$ to be any hybrid kernel function for which the maximum is achieved in (3.1). We now state the dual characterization of the threshold degree.

NECESSARY AND SUFFICIENT CONDITION 3.3. Let $f:\{0,1\}^{n} \rightarrow\{-1,+1\}$ be given, $d=\operatorname{deg}_{ \pm}(f)$. Then there is a distribution $\mu$ over $\{0,1\}^{n}$ with

$$
\underset{x \boxminus \mu}{\mathbf{E}}\left[f(x) X_{S}(x)\right]=0 \quad(|S|<d) .
$$

Alternately, it can be derived as a necessary condition to Necessary and sufficient condition 3.1. We close this section with one final dual characterization, corresponding to finite string representation by integer polynomials.

NECESSARY AND SUFFICIENT CONDITION 3.4. Fix a hybrid kernel function $f:\{0,1\}^{n} \rightarrow\{-1,+1\}$ and an integer $d \geq \operatorname{deg}_{ \pm}(f)$. Then for every distribution $\mu$ on $\{0,1\}^{n}$,

$$
\max _{|S| \leq d}\left|\underset{x \Xi \mu}{\mathbf{E} \mu}\left[f(x) X_{S}(x)\right]\right| \geq \frac{1}{W(f, d)} .
$$

Furthermore, there exists a distribution $\mu$ such that

$$
\max _{|S| \leq d}\left|\underset{x \boxminus \mu}{\mathbf{E}}\left[f(x) X_{S}(x)\right]\right| \leq\left(\frac{2 n}{W(f, d)}\right)^{1 / 2} .
$$

\section{PRELIMINARY IMPACT CRITERIA: II}

We now turn to the second ingredient of our proof, a certain family of real rectangular array of inverted Index summarization expressions that we introduced. Our goal here is to explicitly calculate their singular key-values. As we shall see later, this provides a convenient means to generate hard resizable Hadoop cluster problems.

Let $t$ and $n$ be positive integers, where $t<n$ and $t \mid n$. Partition $[n]$ into $t$ contiguous blocks, each with $n / t$ elements: 


$$
\begin{gathered}
{[n]=\left\{1,2, \ldots, \frac{n}{t}\right\} \cup\left\{\frac{n}{t}+1, \ldots, \frac{2 n}{t}\right\} \cup \ldots} \\
\cup\left\{\frac{(t-1) n}{t}+1, \ldots, n\right\} .
\end{gathered}
$$

Let $\mathrm{V}(n, t)$ denote the family of subsets $V \subseteq[n]$ that have exactly one element in each of these blocks (in particular, $|V|=t)$. Clearly, $|\mathrm{V}(n, t)|=(n / t)^{t}$. For a file finite string $x \in\{0,1\}^{n}$ and a set $V \in \mathrm{V}(n, t)$, define the projection of $x$ onto $V$ by

$$
\left.x\right|_{V}=\left(x_{i 1}, x_{i 2}, \ldots, x_{i t}\right) \in\{0,1\}^{t},
$$

where $i_{1}<i_{2}<\cdots<i_{t}$ are the elements of $V$. We are ready for a formal intention of our rectangular array of inverted Index summarization expressions family.

INTENTION 4.1. For $\phi:\{0,1\}^{t} \rightarrow \mathbb{R}$, the $(n, t, \phi)$-template matching rectangular array of inverted Index summarization expressions is the real rectangular array of inverted Index summarization expressions $A$ given by

$$
A=\left[\phi\left(\left.x\right|_{V} \oplus w\right)\right]_{x \in\{0,1\}^{n},(V, w) \in V(n, t) \times\{0,1\}^{t}} .
$$

In words, $A$ is the rectangular array of inverted Index summarization expressions of size $2^{n}$ by $(n / t)^{t} 2^{t}$ whose rows are indexed by finite strings $x \in\{0,1\}^{n}$, whose columns are indexed by pairs $(V, w) \in \mathrm{V}(n, t) \times\{0,1\}^{t}$, and whose entries are given by $A_{x,(V, w)}=\phi\left(\left.x\right|_{V} \oplus w\right)$.

The logic behind the term "template matching rectangular array of inverted Index summarization expressions" is as follows: a mosaic arises from repetitions of a template matching in the same way that $A$ arises from applications of $\phi$ to various subsets of the variables. Our approach to analyzing the singular key-values of a template matching rectangular array of inverted Index summarization expressions $A$ will be to represent it as the sum of simpler rectangular array of inverted Index summarization expressions and analyze them instead. For this to work, we should be able to reconstruct the singular keyvalues of $A$ from those of the simpler rectangular array of inverted Index summarization expressions. Just when this can be done is the subject of the following sufficient condition.

SUFFICIENT CONDITION 4.2. Let $A, B$ be real rectangular array of inverted Index summarization expressions with $A B^{\mathrm{T}}=0$ and $A^{\mathrm{T}} B=0$. Then the nonzero singular key-values of $A+B, \quad$ counting multiplicities, are $\sigma_{1}(A), \ldots, \sigma_{\mathrm{rk} A}(A), \sigma_{1}(B), \ldots, \sigma_{\mathrm{rk} B}(B)$.

Proof. The claim is trivial when $A=0$ or $B=0$, so assume otherwise. Since the singular key-values of $A+B$ are precisely the square roots of the key-values of $(A+$ $B)(A+B)^{\mathrm{T}}$, it suffices to compute the spectrum of the latter rectangular array of inverted Index summarization expressions. Now,

$$
\begin{aligned}
(A+B)(A+B)^{\mathrm{T}} & =A A^{\mathrm{T}}+B B^{\mathrm{T}}+\underbrace{A B^{\mathrm{T}}}_{=0}+\underbrace{B A^{\mathrm{T}}}_{=0} \\
& =A A^{\mathrm{T}}+B B^{\mathrm{T}} .
\end{aligned}
$$

Fix spectral decompositions

$$
A A^{\mathrm{T}}=\sum_{i=1}^{\mathrm{rk} A} \sigma_{i}(A)^{2} u_{i} u_{i}^{\mathrm{T}}, \quad B B^{\mathrm{T}}=\sum_{j=1}^{\mathrm{rk} B} \sigma_{j}(B)^{2} v_{j} v_{j}^{\mathrm{T}} .
$$

Then

$$
\begin{aligned}
& \sum_{i=1}^{\mathrm{rk} A} \sum_{j=1}^{\mathrm{rk} B} \sigma_{i}(A)^{2} \sigma_{j}(B)^{2}\left\langle u_{i}, v_{j}\right\rangle^{2} \\
& \quad=\left\langle\sum_{i=1}^{\mathrm{rk} A} \sigma_{i}(A)^{2} u_{i} u_{i}^{\mathrm{T}}, \sum_{j=1}^{\mathrm{rk} B} \sigma_{j}(B)^{2} v_{j} v_{j}^{\mathrm{T}}\right| \\
& =\left\langle A A^{\mathrm{T}}, B B^{\mathrm{T}}\right\rangle \\
& =\operatorname{tr}\left(A A^{\mathrm{T}} B B^{\mathrm{T}}\right) \\
& =\operatorname{tr}\left(A \cdot 0 \cdot B^{\mathrm{T}}\right) \\
& =0 .
\end{aligned}
$$

Since $\sigma_{i}(A) \sigma_{j}(B)>0$ for all $i, j$, it follows from (4.2) that $\left\langle u_{i}, v_{j}\right\rangle=0$ for all $i, j$. Put differently, the vectors $u_{1}, \ldots, u_{\mathrm{rk} A}$, $v_{1}, \ldots, v_{\mathrm{rk} B}$ form an orthonormal set. Recalling (4.1), we conclude that the spectral decomposition of $(A+B)(A+B)^{\mathrm{T}}$ is

$$
\sum_{i=1}^{\text {rk } A} \sigma_{i}(A)^{2} u_{i} u_{i}^{\mathrm{T}}+\sum_{j=1}^{\mathrm{rk} B} \sigma_{j}(B)^{2} v_{j} v_{j}^{\mathrm{T}}
$$

and thus the nonzero key-values of $(A+B)(A+B)^{\mathrm{T}}$ are as claimed.

We are ready for the main result of this section.

NECESSARY AND SUFFICIENT CONDITION 4.3. Let $\phi:\{0,1\}^{t} \rightarrow \mathbb{R}$ be given. Let $A$ be the $(n, t, \phi)$-template matching rectangular array of inverted Index summarization expressions. Then the nonzero singular key-values of $A$, counting multiplicities, are:

$$
\bigcup_{S: \widehat{\phi}(S) \neq 0}\left\{\sqrt{2^{n+t}\left(\frac{n}{t}\right)^{t}}\right.
$$

$\cdot|\hat{\phi}(S)|\left(\frac{t}{n}\right)^{|S| / 2}, \quad$ repeated $\left(\frac{n}{t}\right)^{|S|}$ times $\}$.

In particular,

$$
\|A\|=\sqrt{2^{n+t}\left(\frac{n}{t}\right)^{t}} \max _{S \subseteq[t]}\left\{|\hat{\phi}(S)|\left(\frac{t}{n}\right)^{|S| / 2}\right\} .
$$

Proof. For each $S \subseteq[t]$, let $A_{S}$ be the $\left(n, t, X_{S}\right)$-template matching rectangular array of inverted Index summarization expressions. Thus,

$$
A=\sum_{S \subseteq[t]} \hat{\phi}(S) A_{S} .
$$

Fix arbitrary $S, T \subseteq[t]$ with $S \neq T$. Then

$$
A_{S} A_{T}^{\mathrm{T}}=\left[\sum_{V \in V(n, t)} \sum_{w \in\{0,1\}^{t}} X_{S}\left(\left.x\right|_{V} \oplus w\right) X_{T}\left(\left.y\right|_{V} \oplus w\right)\right]_{x, y}
$$

$=[\sum_{V \in V(n, t)} X_{S}\left(\left.x\right|_{V}\right) X_{T}\left(\left.y\right|_{V}\right) \underbrace{\sum_{w \in\{0,1\}^{t}} X_{S}(w) X_{T}(w)}_{=0}]_{x, y}$ 
$=0$.

$$
Q_{1 / 7}^{*}(F)>\frac{1}{4} \operatorname{deg}_{1 / 3}(f) \log \left(\frac{n}{t}\right)-3 .
$$

Similarly,

$$
=[X_{S}(w) X_{T}\left(w^{\prime}\right) \underbrace{\sum_{x \in\{0,1\}^{n}} X_{S}\left(\left.x\right|_{V}\right) X_{T}\left(\left.y\right|_{V^{\prime}}\right)}_{=0}]_{(V, w),\left(V^{\prime}, w^{\prime}\right)}=0 .
$$

By (4.3)-(4.5) and Sufficient condition 4.2, the nonzero singular key-values of $A$ are the union of then on zero singular key-values of all $\hat{\phi}(S) A_{S}$, counting multiplicities. Therefore, the proof will be complete once we show that the only nonzero singular key-value of $A_{S}^{\mathrm{T}} A_{S}$ is $2^{n+t}(n / t)^{t-|S|}$, with multiplicity $(n / t)^{|S|}$. It is convenient to write this rectangular array of inverted Index summarization expressions as

$$
\begin{aligned}
A_{S}^{\mathrm{T}} A_{S} & =\left[X_{S}(w) X_{S}\left(w^{\prime}\right)\right]_{w, w^{\prime}} \\
& \otimes\left[\sum_{x \in\{0,1\}^{n}} X_{S}\left(\left.x\right|_{V}\right) X_{S}\left(\left.y\right|_{V^{\prime}}\right)\right]_{V, V^{\prime}} .
\end{aligned}
$$

The first rectangular array of inverted Index summarization expressions in this factorization has PageRank1 and entries \pm 1 , which means that its only nonzero singular key-value is $2^{t}$ with multiplicity 1 . The other rectangular array of inverted Index summarization expressions, call it $M$, is permutation-similar to

$$
2^{n}\left[\begin{array}{llll}
J & & & \\
& J & & \\
& & \ddots & \\
& & & J
\end{array}\right],
$$

where $J$ is the all-ones square rectangular array of inverted Index summarization expressions of order $(n / t)^{t-|S|}$. This means that the only nonzero singular key-value of $M$ is $2^{n}(n / t)^{t-|S|}$ with multiplicity $(n / t)^{|S|}$. It follows from elementary properties of the spectrum of $A_{S}^{\mathrm{T}} A_{S}$ is as claimed.

\section{DESCRIPTIVE STUDY I: I}

The previous two sections examined relevant dual representations and the spectrum of template matching rectangular array of inverted Index summarization expressions. Having studied these notions in their pure and basic form, we now apply our findings to resizable Hadoop cluster's complexity. Specifically, we establish the template matching rectangular array of inverted Index summarization expressions for resizable Hadoop cluster's complexity, which gives strong lower bounds for every template matching rectangular array of inverted Index summarization expressions generated by a hybrid cost function with high approximate degree.

NECESSARY AND SUFFICIENT CONDITION 1.1 (restated). Let $F$ be the $(n, t, f)$-template matching rectangular array of inverted Index summarization expressions, where $f:\{0,1\}^{t} \rightarrow\{-1,+1\}$ is given. Then for every $\epsilon \in[0,1)$ and every $\delta<\epsilon / 2$,

$$
Q_{\delta}^{*}(F) \geq \frac{1}{4} \operatorname{deg}_{\epsilon}(f) \log \left(\frac{n}{t}\right)-\frac{1}{2} \log \left(\frac{3}{\epsilon-2 \delta}\right) .
$$

In particular,
Proof. Since (5.1) immediately implies (5.2), we will focus on the former in the remainder of the proof. Let $d=\operatorname{deg}_{\epsilon}(f) \geq 1$. By Necessary and sufficient condition 3.2, there is a hybrid kernel function $\psi:\{0,1\}^{t} \rightarrow \mathbb{R}$ such that:

$$
\begin{aligned}
& \hat{\psi}(S)=0 \quad(|S|<d), \\
& \sum_{z \in\{0,1\}^{t}}|\psi(z)|=1, \\
& \sum_{z \in\{0,1\}^{t}} \psi(z) f(z)>\epsilon .
\end{aligned}
$$

Let $\psi$ be the $\left(n, t, 2^{-n}(n / t)^{-t} \psi\right)$-template matching rectangular array of inverted Index summarization expressions. Then (5.4) and (5.5) show that

$$
\|\psi\|_{1}=1, \quad\langle F, \psi\rangle>\epsilon .
$$

Our last task is to calculate $\|\psi\|$. By (5.4) and Necessary and sufficient condition 2.1,

$$
\max _{S \subseteq[t]}|\hat{\psi}(S)| \leq 2^{-t} .
$$

Necessary and sufficient condition 4.3 yields, in view of (5.3) and (5.7):

Now (5.1) follows from (5.6), (5.8), and Necessary and sufficient condition 2.8 .

Necessary and sufficient condition 1.1 gives lower bounds not only for bounded-capacity resizable Hadoop cluster but also for resizable Hadoop cluster's preference limitation protocols with capacity probability $\frac{1}{2}-o(1)$. For example, if a hybrid kernel function $f:\{0,1\}^{t} \rightarrow\{-1,+1\}$ requires a polynomial of degree $d$ for approximation within $1-o(1)$, equation (5.1) gives a lower bound for small-bias resizable Hadoop cluster. We will complement and refine that estimate in the next section, which is dedicated to small-bias resizable Hadoop cluster.

We now prove the necessary condition to Necessary and sufficient condition 1.1 on hybrid kernel function composition, stated in the inception.

Proof of Necessary condition 1.2. The $(2 t, t, f)$-template matching rectangular array of inverted Index summarization expressions occurs as a subset of rectangular array of inverted Index summarization expressions of $[F(x, y)]_{x, y \in\{0,1\}^{4 t}}$.

Finally, we show that the lower bound (5.2) derived above for bounded-capacity resizable Hadoop cluster's complexity is tight up to a polynomial factor, even for deterministic preference limitation protocols.

NECESSARY AND SUFFICIENT CONDITION 5.1. Let $F$ be the $(n, t, f)$-template matching rectangular array of inverted Index summarization expressions, where $f:\{0,1\}^{t} \rightarrow$ $\{-1,+1\}$ is given. Then

$D(F) \leq O(\operatorname{dt}(f) \log (n / t)) \leq O\left(\operatorname{deg}_{1 / 3}(f)^{6} \log (n / t)\right)$,

where $\operatorname{dt}(f)$ is the least depth of a decision tree for $f$. In particular, (5.2) is tight upto a polynomial factor.

$$
\|\psi\| \leq\left(\frac{t}{n}\right)^{d / 2}\left(2^{n+t}\left(\frac{n}{t}\right)^{t}\right)^{-1 / 2} .
$$


Proof. That $\operatorname{dt}(f) \leq O\left(\operatorname{deg}_{1 / 3}(f)^{6}\right)$ for all hybrid cost functions $f$. Therefore, it suffices to prove an upper bound of $O(d \log (n / t))$ on the deterministic resizable Hadoop cluster's complexity of $F$, where $d=\operatorname{dt}(f)$.

The needed deterministic preference limitation protocol is not well known. Fix a depth- $d$ decision tree for $f$. Let $(x,(V, w))$ be a given input. Alice and Bob start at the root of the decision tree, labeled by some variable $i \in\{1, \ldots, t\}$. By exchanging $\lceil\log (n / t)\rceil+2$ compatible JAR files, Alice and Bob determine $\left(\left.x\right|_{V}\right)_{i} \oplus w_{i} \in\{0,1\}$ and take the corresponding branch of thetree. The process repeats until a leaf is reached, at which point both parties learn $f\left(\left.x\right|_{V} \oplus w\right)$.

\section{DESCRIPTIVE STUDY I: II}

As we have already mentioned, Necessary and sufficient condition 1.1 of the previous section can be used to obtain lower bounds not only for bounded-capacity resizable Hadoop cluster but also small-bias resizable Hadoop cluster. In the latter case, one first needs to show that the base hybrid kernel function $f:\{0,1\}^{t} \rightarrow\{-1,+1\}$ cannot be approximated point wise within $1-o(1)$ by a real polynomial of a given degree $d$. In this section, we derive a different lower bound for small-bias resizable Hadoop cluster, this time using the assumption that the threshold weight $W(f, d)$ is high. We will see that this new lower bound is nearly optimal and closely related to the lower bound in Necessary and sufficient condition 1.1.

NECESSARY AND SUFFICIENT CONDITION 6.1. Let $F$ be the $(n, t, f)$-template matching rectangular array of inverted Index summarization expressions, where $f:\{0,1\}^{t} \rightarrow$ $\{-1,+1\}$ is given. Then for every integer $d \geq 1$ and real $\gamma \in(0,1)$,

$$
\begin{aligned}
& Q_{1 / 2-\gamma / 2}^{*}(F) \\
& \quad \geq \frac{1}{4} \min \left\{d \log \frac{n}{\mathrm{t}}, \log \frac{W(f, d-1)}{2 t}\right\} \\
& \quad-\frac{1}{2} \log \frac{3}{\gamma} .
\end{aligned}
$$

In particular,

$$
Q_{1 / 2-\gamma / 2}^{*}(F) \geq \frac{1}{4} \operatorname{deg}_{ \pm}(f) \log \left(\frac{n}{\mathrm{t}}\right)-\frac{1}{2} \log \frac{3}{\gamma} .
$$

Proof. Letting $d=\operatorname{deg}_{ \pm}(f)$ in (6.1) yields (6.2), since $W(f, d-1)=\infty$ in that case. In the remainder of the proof, we focus on (6.1) alone.

We claim that there exists a distribution $\mu$ on $\{0,1\}^{t}$ such that

$$
\max _{|S|<d}\left|\underset{z \boxminus \mu}{\mathbf{E} \mu}\left[f(z) X_{S}(z)\right]\right| \leq\left(\frac{2 t}{W(f, d-1)}\right)^{1 / 2} .
$$

For $d \leq \operatorname{deg}_{ \pm}(f)$, the claim holds by Necessary and sufficient condition 3.3 since $W(f, d-1)=\infty$ in that case.

For $d>\operatorname{deg}_{ \pm}(f)$, the claim holds by Necessary and sufficient condition 3.4 .

Now, define $\psi:\{0,1\}^{t}$ by $\psi(z)=f(z) \mu(z)$. It follows from (6.3) that

$$
\begin{aligned}
& |\hat{\psi}(S)| \leq 2^{-t}\left(\frac{2 t}{W(f, d-1)}\right)^{1 / 2}(|S|<d), \\
& \sum_{z \in\{0,1\}^{t}}|\psi(z)|=1
\end{aligned}
$$

$$
\sum_{z \in\{0,1\}^{t}} \psi(z) f(z)=1
$$

Let $\psi$ be the $\left(n, t, 2^{-n}(n / t)^{-t} \psi\right)$-template matching rectangular array of inverted Index summarization expressions. Then (6.5) and (6.6) show that

$$
\|\psi\|_{1}=1, \quad\langle F, \psi\rangle=1 .
$$

It remains to calculate $\|\psi\|$. By (6.5) and Necessary and sufficient condition 2.1,

$$
\max _{S \subseteq[t]}|\hat{\psi}(S)| \leq 2^{-t} .
$$

Necessary and sufficient condition 4.3 yields, in view of (6.4) and (6.8):

(6.9) $\|\psi\|$

$$
\leq \max \left\{\left(\frac{t}{n}\right)^{d / 2},\left(\frac{2 t}{W(f, d-1)}\right)^{1 / 2}\right\}\left(2^{n+t}\left(\frac{n}{t}\right)^{t}\right)^{-1 / 2} \text {. }
$$

Now (6.1) follows from (6.7), (6.9), and Necessary and sufficient condition 2.8 .

Recall from Necessary and sufficient condition 2.5 that the quantities $E(f, d)$ and $W(f, d)$ are related for all $f$ and $d$. In particular, the lower bounds for small-bias resizable Hadoop cluster in Propositions 1.1 and 6.1 are quite close, and either one can be approximately deduced from the other. In deriving both findings from scratch, as we did, our motivation was to obtain the tightest bounds and to illustrate the template matching rectangular array of inverted Index summarization expressions in different contexts. We will now see that the lower bound in Necessary and sufficient condition 6.1 is close to optimal, even for channel preference limitation protocols.

NECESSARY AND SUFFICIENT CONDITION 6.2. Let $F$ be the $(n, t, f)$-template matching rectangular array of inverted Index summarization expressions, where $f:\{0,1\}^{t} \rightarrow$ $\{-1,+1\}$ is given. Then for every integer $d \geq \operatorname{deg}_{ \pm}(f)$,

$$
\begin{aligned}
& Q_{1 / 2-\gamma / 2}^{*}(F) \leq R_{1 / 2-\gamma / 2}(F) \leq d \log \left(\frac{n}{\mathrm{t}}\right)+3, \\
& \text { where } \gamma-1 / W(f, d) .
\end{aligned}
$$

Proof. The resizable Hadoop cluster's preference limitation protocol that we will describe is standard. Put $W=W(f, d)$ and fix a representation

$$
f(z) \equiv \operatorname{sgn}\left(\sum_{S \subseteq[t],|S| \leq d} \lambda_{S} X_{S}(z)\right),
$$

where the integers $\lambda_{S}$ satisfy $\sum\left|\lambda_{S}\right|=W$. On input $(x,(V, w))$, the preference limitation protocol proceedsas follows. Let $i_{1}<i_{2}<\cdots<i_{t}$ be the elements of $V$. Alice and Bob use their shared randomness to pick a set $S \subseteq[t]$ with $|S| \leq d$, according to the probability distribution $\left|\lambda_{S}\right| / W$. Next, Bob sends Alice the indices $\left\{i_{j}: j \in S\right\}$ as well as the file $X_{S}(w)$. With this information, Alice computes the product $\operatorname{sgn}\left(\lambda_{S}\right) X_{S}\left(\left.x\right|_{V}\right) X_{S}(w)=\operatorname{sgn}\left(\lambda_{S}\right) X_{S}\left(\left.x\right|_{V} \oplus w\right)$ and announces the result as the output of the preference limitation protocol.

Assuming an optimal encoding of the compatible JAR files, the resizable Hadoop cluster's cost of this preference limitation protocol is bounded by

$$
\left\lceil\log \left(\frac{n}{t}\right)^{d}\right\rceil+2 \leq d \log \left(\frac{n}{t}\right)+3
$$


as desired. On each input $x, V, w$, the output of the preference limitation protocol is a random variable that $P(x, V, w) \in$ $\{-1,+1\}$ obeys

$$
\begin{aligned}
& f\left(\left.x\right|_{V} \oplus w\right) \mathbf{E}[P(x, V, w)] \\
& =f\left(\left.x\right|_{V} \oplus w\right) \sum_{|S| \leq d} \frac{\left|\lambda_{S}\right|}{W} \operatorname{sgn}\left(\lambda_{S}\right) X_{S}\left(\left.x\right|_{V}\right. \\
& \oplus w) \\
& =\frac{1}{W}\left|\sum_{|S| \leq d} \lambda_{S} X_{S}\left(\left.x\right|_{V} \oplus w\right)\right| \\
& \geq \frac{1}{W}
\end{aligned}
$$

which means that the preference limitation protocol produces the correct answer with probability $\frac{1}{2}+\frac{1}{2 W}$ or greater.

\section{PRESCRIPTIVE STUDY: I}

We now restate some of the findings of the previous section in terms of discrepancy, a key notion already mentioned in Section 2.4. This quantity figures prominently in the study of small-bias resizable Hadoop cluster as well as various applications, such as inference theory and cluster complexity [36].

For a hybrid cost function $f: X \times Y \rightarrow\{-1,+1\}$ and a probability distribution $\lambda$ on $X \times Y$, the discrepancy of $f$ under $\lambda$ is defined by

$$
\operatorname{disc}_{\lambda}(f)=\max _{\substack{S \subseteq X \\ T \subseteq Y}}\left|\sum_{x \in S} \sum_{y \in T} \lambda(x, y) f(x, y)\right| .
$$

We put

$$
\operatorname{disc}(f)=\min _{\lambda} \operatorname{disc}_{\lambda}(f) .
$$

As usual, we will identify a hybrid kernel function $f: X \times Y \rightarrow$ $\{-1,+1\}$ with its resizable Hadoop cluster rectangular array of inverted Index summarization expressions $F=$ $[f(x, y)]_{x, y}$ and use the conventions $\operatorname{disc}_{\lambda}(F)=\operatorname{disc}_{\lambda}(f)$ and $\operatorname{disc}(F)=\operatorname{disc}(f)$.

The above intention of discrepancy is not convenient to work with, and we will use a well-known rectangular array of inverted Index summarization expressions-analytic reformulation. For rectangular array of inverted Index summarization expressions $A=\left[A_{x y}\right]$ and $B=\left[B_{x y}\right]$, recall that their product is given by $A \circ B=\left[A_{x y} B_{x y}\right]$.

NECESSARY AND SUFFICIENT CONDITION 7.1. Let $X, Y$ be finite information sets, $f: X \times Y \rightarrow\{-1,+1\}$ a given hybrid kernel function. Then

$$
\operatorname{disc}_{P}(f) \leq \sqrt{|X||Y|}\|P \circ F\|,
$$

where $F=[f(x, y)]_{x \in X, y \in Y}$ and $P$ is any rectangular array of inverted Index summarization expressions whose entries are nonnegative and sum to 1 (viewed as a probability distribution). In particular,

$$
\operatorname{disc}(f) \leq \sqrt{|X||Y|} \min _{P}\|P \circ F\|,
$$

where the minimum is over rectangular array of inverted Index summarization expressions $P$ whose entries are nonnegative and sum to 1 .
Proof. We have

$$
\begin{aligned}
& \operatorname{disc}_{P}(f)=\max _{S, T}\left|\mathbf{1}_{S}^{\mathrm{T}}(P \circ F) \mathbf{1}_{T}\right| \\
& \leq \max _{S, T}\left\{\left\|\mathbf{1}_{S}\right\| \cdot\|P \circ F\| \cdot\left\|\mathbf{1}_{T}\right\|\right\} \\
& \quad=\|P \circ F\| \sqrt{|X||Y|},
\end{aligned}
$$

as claimed.

We will need one last ingredient, a well-known lower bound on resizable Hadoop cluster's complexity in terms of discrepancy.

NECESSARY AND SUFFICIENT CONDITION 7.2. For every hybrid kernel functionf : $X \times Y \rightarrow\{-1,+1\}$ and every $\gamma \in(0,1)$,

$$
R_{1 / 2-\gamma / 2}(f) \geq \log \frac{\gamma}{\operatorname{disc}(f)} .
$$

Using Propositions 6.1 and 6.2, we will now characterize the discrepancy of template matching rectangular array of inverted Index summarization expressions in terms of threshold weight.

NECESSARY AND SUFFICIENT CONDITION 7.3. Let $F$ be the $(n, t, f)$-template matching rectangular array of inverted Index summarization expressions, where $f:\{0,1\}^{t} \rightarrow\{-1,+1\}$ is given. Then for every integer $d \geq 0$,

$$
\operatorname{disc}(F) \geq \frac{1}{8 W(f, d)}\left(\frac{t}{n}\right)^{d}
$$

and

$$
\operatorname{disc}(F)^{2} \leq \max \left\{\frac{2 t}{W(f, d-1)},\left(\frac{t}{n}\right)^{d}\right\} .
$$

In particular,

$$
\operatorname{disc}(F) \leq\left(\frac{t}{n}\right)^{\operatorname{deg}_{ \pm}(f) / 2}
$$

Proof. The lower bound (7.1) is immediate from Necessary and sufficient condition 6.2 and Necessary and sufficient condition 7.2. For the upper bound (7.2), construct the rectangular array of inverted Index summarization expressions $\psi$ as in the proof of Necessary and sufficient condition 6.1. Then (6.7) shows that $\psi=F \circ P$ for a nonnegative rectangular array of inverted Index summarization expressions $P$ whose entries sum to 1 . As a result, (7.2) follows from (6.9) and Necessary and sufficient condition 7.1. Finally, (7.3) follows by taking $d=\operatorname{deg}_{ \pm}(f)$ in (7.2), since $W(f, d-1)=\infty$ in that case.

This settles Necessary and sufficient condition 1.5 from the inception. Necessary and sufficient condition 7.3 follows up and considerably improves on the Degree/Discrepancy Necessary and sufficient condition.

NECESSARY AND SUFFICIENT CONDITION 7.4. Let $f:\{0,1\}^{t} \rightarrow\{-1,+1\}$ be given. Fix an integer $n \geq t$. Let $M=\left[f\left(\left.x\right|_{S}\right)\right]_{x, S}$, where the row index $x$ ranges over $\{0,1\}^{n}$ and the column index $S$ ranges over all t-element subsets of $\{1,2, \ldots, n\}$. Then

$$
\operatorname{disc}(M) \leq\left(\frac{4 e t^{2}}{n \operatorname{deg}_{ \pm}(f)}\right)^{\operatorname{deg}_{ \pm}(f) / 2} .
$$

Note that (7.3) is already stronger than Necessary and sufficient condition 7.4. In Section 10, we will see an example when 
Necessary and sufficient condition 7.3 gives an exponential improvement on Necessary and sufficient condition 7.4.

Threshold weight is typically easier to analyze than the approximate degree. For completeness, however, we will now supplement Necessary and sufficient condition 7.3 with an alternate bound on the discrepancy of a template matching rectangular array of inverted Index summarization expressions in terms of the approximate degree.

NECESSARY AND SUFFICIENT CONDITION 7.5. Let $F$ be the $(n, t, f)$-template matching rectangular array of inverted Index summarization expressions, for a given hybrid kernel function $f:\{0,1\}^{t} \rightarrow\{-1,+1\}$. Then for every $\gamma>0$,

$$
\operatorname{disc}(F) \leq \gamma+\left(\frac{t}{n}\right)^{\operatorname{deg}_{ \pm}(f) / 2}
$$

Proof. Let $d=\operatorname{deg}_{1-\gamma}(f) \geq 1$. Define $\epsilon=1-\gamma$ and construct the rectangular array of inverted Index summarization expressions $\psi$ as in the proof of Necessary and sufficient condition 1.1. Then (5.6) shows that $\psi=H \circ P$, where $H$ is a finite string rectangular array of inverted Index summarization expressions and $P$ is a nonnegative rectangular array of inverted Index summarization expressions whose entries sum to 1. Viewing $P$ as a probabilitydistribution, we infer from (5.8) and Necessary and sufficient condition 7.1 that

$$
\operatorname{disc}_{P}(H) \leq\left(\frac{t}{n}\right)^{d / 2}
$$

Moreover,

$$
\begin{aligned}
\operatorname{disc}_{P}(F) & \leq \operatorname{disc}_{P}(H)+\|(F-H) \circ P\|_{1} \\
& =\operatorname{disc}_{P}(H)+1-\langle F, H \circ P\rangle \\
& \leq \operatorname{disc}_{P}(H)+\gamma,
\end{aligned}
$$

where the last step follows because $\langle F, \psi\rangle>\epsilon=1-\gamma$ by (5.6). The proof is complete in view of (7.4) and (7.5).

\section{PRESCRIPTIVE STUDY: II}

We will now use the findings of the previous sections to analyze the approximate PageRank and approximate trace distance norm of template matching rectangular array of inverted Index summarization expressions. These notions were originally motivated by lower bounds on resizable Hadoop cluster. However, they also arise in inference theory and are natural analytic quantities in their own right.

NECESSARY AND SUFFICIENT CONDITION 8.1. Let $F$ be the $(n, t, f)$-template matching rectangular array of inverted Index summarization expressions, where $f:\{0,1\}^{t} \rightarrow\{-1,+1\}$ is given. Let $s=2^{n+t}(n / t)^{t}$ be the number of entries in $F$. Then for every $\epsilon \in[0,1)$ and every $\delta \in[0, \epsilon]$,

$$
\|F\|_{\Sigma, \delta} \geq(\epsilon-\delta)\left(\frac{n}{t}\right)^{\operatorname{deg}_{\epsilon}(f) / 2} \sqrt{s}
$$

and

$$
\operatorname{rk}_{\delta} F \geq\left(\frac{\epsilon-\delta}{1+\delta}\right)^{2}\left(\frac{n}{t}\right)^{\operatorname{deg}_{\epsilon}(f)} .
$$

Proof. We may assume that $\operatorname{deg}_{\epsilon}(f) \geq 1$, since otherwise $f$ is a constant hybrid kernel function and the claims hold trivially by taking $\psi=F$ in Necessary and sufficient condition 2.2. Construct $\psi$ asin the proof of Necessary and sufficient condition 1.1. Then the claimed lower bound on $\|F\|_{\sum, \delta}$ follows from (5.6), (5.8), and Necessary and sufficient condition 2.2. Finally, (8.2) follows immediately from (8.1) and Necessary and sufficient condition 2.3.

We prove an additional lower bound in the case of small-bias approximation.

NECESSARY AND SUFFICIENT CONDITION 8.2. Let $F$ be the $(n, t, f)$-template matching rectangular array of inverted Index summarization expressions, where $f:\{0,1\}^{t} \rightarrow\{-1,+1\}$ is given. Let $s=2^{n+t}(n / t)^{t}$ be the number of entries in $F$. Then for every $\gamma \in(0,1)$ and every integer $d \geq 1$,

(8.3) $\|F\|_{\Sigma, 1-\gamma} \geq \gamma \min \left\{\left(\frac{n}{t}\right)^{d / 2},\left(\frac{W(f, d-1)}{2 t}\right)^{1 / 2}\right\} \sqrt{s}$

and

$$
\mathrm{rk}_{1-\gamma} F \geq\left(\frac{\gamma}{2-\gamma}\right)^{2} \min \left\{\left(\frac{n}{t}\right)^{d}, \frac{W(f, d-1)}{2 t}\right\}
$$

In particular,

$$
\|F\|_{\sum, 1-\gamma} \geq \gamma\left(\frac{n}{t}\right)^{\operatorname{deg}_{ \pm}(f) / 2} \sqrt{s}
$$

and

$$
\mathrm{rk}_{1-\gamma} F \geq\left(\frac{\gamma}{2-\gamma}\right)^{2}\left(\frac{n}{t}\right)^{\operatorname{deg}_{ \pm}(f)} .
$$

Proof. Construct $\psi$ as in the proof of Necessary and sufficient condition 6.1. Then the claimed lower boundon $\|F\|_{\Sigma, \delta}$ follows from (6.7), (6.9), and Necessary and sufficient condition 2.2. Now (8.4) follows from (8.3) and Necessary and sufficient condition 2.3. Finally, (8.5) and (8.6) follow by taking $d=$ $\operatorname{deg}_{ \pm}(f)$ in (8.3) and (8.4), respectively, since $W(f, d-1)=$ $\infty$ in that case.

Propositions 8.1 and 8.2 settle Necessary and sufficient condition 1.4 from the inception.

Recall that Necessary and sufficient condition 4.3 gives an easy way to calculate the trace distance norm and PageRank of a template matching rectangular array of inverted Index summarization expressions. In particular, it is straightforward to verify that the lower bounds in (8.2) and (8.4) are close to optimal for various choices of $\epsilon, \delta, \gamma$. For example, one has $\|F-A\|_{\infty} \leq 1 / 3$ by taking $F$ and $A$ to be the $(n, t, f)$-and $(n, t, \phi)$-template matching rectangular array of inverted Index summarization expressions, where $\phi:\{0,1\}^{t} \rightarrow \mathbb{R}$ is any polynomial of degree $\operatorname{deg}_{1 / 3}(f)$ with $\|F-\phi\|_{\infty} \leq 1 / 3$.

\section{DESCRIPTIVE STUDY II: I}

As an illustrative application of the template matching rectangular array of inverted Index summarization expressions, we now give a short and elementary proof of optimal lower bounds for every conjunctive predicate $\quad D:\{0,1, \ldots, n\} \rightarrow$ $\{-1,+1\}$. We first solve the problem for all conjunctive predicates $D$ that change value close to 0 . Extension to the general case will require an additional step.

NECESSARY AND SUFFICIENT CONDITION 9.1. Let $D:\{0,1, \ldots, n\} \rightarrow\{-1,+1\}$ be a given conjunctive predicate. Suppose that $D(\ell) \neq D(\ell-1)$ for some $\ell \leq \frac{1}{8} n$. Then

$$
Q_{1 / 3}^{*}(D) \geq \Omega(\sqrt{n \ell}) .
$$


Proof. It suffices to show that $Q_{1 / 7}^{*}(D) \geq \Omega(\sqrt{n \ell})$. Define $f:\{0,1\}^{\mid n / 4\rfloor} \rightarrow\{-1,+1\} \quad$ by $\quad f(z)=D(|z|)$. Then $\operatorname{deg}_{1 / 3}(f) \geq \Omega(\sqrt{n \ell})$ by Necessary and sufficient condition 2.6. Necessary and sufficient condition 1.1 implies that

$$
Q_{1 / 7}^{*}(F) \geq \Omega(\sqrt{n \ell}),
$$

where $F$ is the $(2\lfloor n / 4\rfloor,\lfloor n / 4\rfloor, f)$-template matching rectangular array. Since $F$ occurs as a subset of rectangular array of inverted Index summarization expressions of $[D(\mid x \wedge$ $y \mid)]_{x, y}$, the proof is complete.

The remainder of this section is a simple if tedious exercise in shifting and padding. We note that proof concludes in a similar way.

NECESSARY AND SUFFICIENT CONDITION 9.2. Let $D:\{0,1, \ldots, n\} \rightarrow\{-1,+1\}$ be a given conjunctive predicate. Suppose that $D(\ell) \neq D(\ell-1)$ for some $\ell \leq \frac{1}{8} n$. Then

$$
Q_{1 / 3}^{*}(D) \geq c(n-\ell)
$$

for some absolute constant $c>0$.

Proof. Consider the resizable Hadoop cluster problem of computing $D(|x \wedge y|)$ when the last $k$ compatible JAR files in $x$ and $y$ are fixed to 1 . In other words, the new problem is to compute $D_{k}\left(\left|x^{\prime} \wedge y^{\prime}\right|\right)$ where $x^{\prime}, y^{\prime} \in\{0,1\}^{n-k}$ and the conjunctive predicate $D_{k}:\{0,1, \ldots, n-k\} \rightarrow\{-1,+1\}$, is given by $D_{k}(i) \equiv D(k+i)$. Since the new problem is a restricted version of the original, we have

$$
Q_{1 / 3}^{*}(D) \geq Q_{1 / 3}^{*}\left(D_{k}\right) .
$$

We complete the proof by placing a lower bound on $Q_{1 / 3}^{*}\left(D_{k}\right)$ for

$$
k=\ell-\left\lfloor\frac{\alpha}{1-\alpha} \cdot(n-\ell)\right\rfloor,
$$

where $\alpha=\frac{1}{8}$. Note that $k$ is an integer between 1 and $\ell$ (because $\ell>\alpha n$ ). The equality $k=\ell$ occurs if and only if $\left|\frac{\alpha}{1-\alpha}(n-\ell)\right|=0$, in which case (9.1) holds trivially for $c$ suitably small. Thus, we can assume that $1 \leq k \leq \ell-1$, in which case $D_{k}(\ell-k) \neq D_{k}(\ell-k-1)$ and $\ell-k \leq \alpha(n-$ $k)$. Therefore, Necessary and sufficient condition 9.1 is applicable to $D_{k}$ and yields:

$$
Q_{1 / 3}^{*}\left(D_{k}\right) \geq C \sqrt{(n-k)(\ell-k)},
$$

where $C>0$ is an absolute constant. Calculations reveal:

$$
\begin{aligned}
& n-k=\left\lfloor\frac{1}{1-\alpha} \cdot(n-\ell)\right\rfloor, \\
& \quad \ell-k=\left\lfloor\frac{\alpha}{1-\alpha} \cdot(n-\ell)\right\rfloor .
\end{aligned}
$$

The Necessary and sufficient condition is now immediate from (9.2)-(9.4).

Together, Propositions 9.1 and 9.2 give the main result of this section:

NECESSARY AND SUFFICIENT CONDITION 1.3 (restated from p. 3). Let $D:\{0,1, \ldots, n\} \rightarrow\{-1,+1\}$. Then

$$
Q_{1 / 3}^{*}(D) \geq \Omega\left(\sqrt{n \ell_{0}(D)}+\ell_{1}(D)\right),
$$

where $\ell_{0}(D) \in\{0,1, \ldots,\lfloor n / 2]\}$ and $\ell_{1}(D) \in\{0,1, \ldots,\lceil n / 2\rceil\}$ are the smallest integers such that $D$ is constant in the range $\left[\ell_{0}(D), n-\ell_{1}(D)\right]$.

Proof. If $\ell_{0}(D) \neq 0$, set $\ell=\ell_{0}(D)$ and note that $D(\ell) \neq$ $D(\ell-1)$ by intention. One of Propositions 9.1 and 9.2 must be applicable, and therefore $Q_{1 / 3}^{*}(D) \geq \min \{\Omega(\sqrt{n \ell}), \Omega(n-\ell)\}$. Since $\ell \leq n / 2$, this simplifies to

$$
Q_{1 / 3}^{*}(D) \geq \Omega\left(\sqrt{n \ell_{0}(D)}\right) .
$$

If $\ell_{1}(D) \neq 0$, set $\ell=n-\ell_{1}(D)+1 \geq n / 2$ and note that $D(\ell) \neq D(\ell-1)$ as before. By Necessary and sufficient condition 9.2 ,

$$
Q_{1 / 3}^{*}(D) \geq \Omega\left(\ell_{1}(D)\right) .
$$

The Necessary and sufficient condition follows from (9.5) and (9.6).

\section{DESCRIPTIVE STUDY II: II}

As another application of the template matching rectangular array of inverted Index summarization expressions, we revisit the discrepancy of $\mathrm{AC}^{0}$, the class of polynomial-size constantdepth Hadoop clusters. Independently, [26] exhibited another hybrid kernel function in $\mathrm{AC}^{0}$ with exponentially small discrepancy. We revisit this discrepancy below, considerably sharpening the bound in [25] and giving a new and simple proof of the bound.

Consider the hybrid kernel function $\mathrm{MP}_{m}:\{0,1\}^{4 \mathrm{~m}^{3}} \rightarrow$ $\{-1,+1\}$ given by

$$
\mathrm{MP}_{m}(x)=\bigvee_{i=1}^{m} \bigwedge_{j=1}^{4 \mathrm{~m}^{2}} x_{i j}
$$

Using this hybrid kernel function and the Degree/Discrepancy Necessary and sufficient condition (Necessary and sufficient condition 7.4), an upper bound of $\exp \left\{-\Omega\left(n^{1 / 5}\right)\right\}$ was derived on the discrepancy of an explicit $\mathrm{AC}^{0}$ cluster $f:\{0,1\}^{n} \times$ $\{0,1\}^{n} \rightarrow\{-1,+1\}$ of depth 3 .

We will now sharpen that bound to $\exp \left\{-\Omega\left(n^{1 / 3}\right)\right\}$.

NECESSARY AND SUFFICIENT CONDITION 1.6 (restated). Let $f(x, y)=\mathrm{MP}_{m}(x \vee y)$. Then

$\operatorname{disc}(f)=\exp \{-\Omega(m)\}$.

Proof. Put $d=\lfloor n / 2\rfloor$. We state that $\operatorname{deg}_{ \pm}\left(\mathrm{MP}_{d}\right) \geq d$. Since the $\left(8 d^{3}, 4 d^{3}, \mathrm{MP}_{d}\right)$-template matching rectangular array of inverted Index summarization expressions is a subset of rectangular array of inverted Index summarization expressions of $[f(x, y)]_{x, y}$, the proof is complete in view of equation (7.3) of Necessary and sufficient condition 7.3.

The ODD-MAX-BOUND hybrid kernel function $\mathrm{OMB}_{n}$ : $\{0,1\}^{n} \rightarrow\{-1,+1\}$, is given by

$$
\operatorname{OMB}_{n}(x)=\operatorname{sgn}\left(1+\sum_{i=1}^{n}(-2)^{i} x_{i}\right) .
$$

It is straightforward to compute $\mathrm{OMB}_{n}$ by a linear-size DNF formula and even a decision list. In particular, $\mathrm{OMB}_{n}$ belongs to the class $\mathrm{AC}^{0}$. 
NECESSARY AND SUFFICIENT CONDITION 10.1 (Chazelle et al.). Let $f(x, y)=\mathrm{OMB}_{n}(x \wedge y)$. Then

$\operatorname{disc}(f)=\exp \left\{-\Omega\left(n^{1 / 3}\right)\right\}$.

Using the celebrated findings of Chazelle's papers, we can give a short alternate proof of this Necessary and sufficient condition.

Proof. Put $m=\lfloor n / 4\rfloor$. Shows that $W\left(\mathrm{OMB}_{m}, \mathrm{~cm}^{1 / 3}\right) \geq$ $\exp \left(\mathrm{cm}^{1 / 3}\right)$ for some absolute constant $c>0$. Since the $\left(2 m, m, \mathrm{OMB}_{m}\right)$-template matching rectangular array of inverted Index summarization expressions is a subset of rectangular array of inverted Index summarization expressions of $[f(x, y)]_{x, y}$, the proof is complete by Necessary and sufficient condition 7.3.

REMARK 10.2. The above proofs illustrate that the characterization of the discrepancy of template matching rectangular array of inverted Index summarization expressions. In particular, the representation (10.1) makes it clear that $\operatorname{deg}_{ \pm}\left(\mathrm{OMB}_{n}\right)=1$ and therefore Necessary and sufficient condition 7.4 cannot yield an upper bound better than $n^{-\Omega(1)}$ on the discrepancy of $\mathrm{OMB}_{n}(x \wedge y)$. Necessary and sufficient condition 7.3, on the otherhand, gives an exponentially better upper bound.It is well-known that the discrepancy of a hybrid kernel function $f$ implies a lower bound on the size of majority Hadoop clusters that compute $f$. Following, we record the consequences of Propositions 1.6 and 10.1 in this regard.

NECESSARY AND SUFFICIENT CONDITION 10.3. Any majority vote of threshold that computes the hybrid kernel function

$$
f(x, y)=\mathrm{MP}_{m}(x \vee y)
$$

has size $\exp \{\Omega(m)\}$. Analogously, any majority vote of threshold that computes the hybrid kernel function

$$
f(x, y)=\mathrm{OMB}_{n}(x \wedge y)
$$

has size $\exp \left\{\Omega\left(n^{1 / 3}\right)\right\}$.

\section{CONCLUSIONS}

In previous sections, we characterized various rectangular array of inverted Index summarization expressions-analytic and combinatorial properties of template matching rectangular array of inverted Index summarization expressions, including their channel and resizable Hadoop cluster's complexity, discrepancy, approximate PageRank, and approximate trace distance norm. We conclude this study with another fact about template matching rectangular array of inverted Index summarization expressions.

We observed that the deterministic resizable Hadoop cluster's complexity of a finite string rectangular array of inverted Index summarization expressions $F$ satisfies $D(F) \geq \log \mathrm{rk} F$. The log-PageRank hypothesis is that this lower bound is always tight up to a polynomial factor, i.e., $D(F) \leq(\log r k F)^{O(1)}+$ $O(1)$. Using the findings of the previous sections, we can givea short proof of this hypothesis in the case of template matching rectangular array of inverted Index summarization expressions.

NECESSARY AND SUFFICIENT CONDITION 11.1. Let $f:\{0,1\}^{t} \rightarrow\{-1,+1\}$ be a given hybrid kernel function, $d=\operatorname{deg}(f)$. Let $F$ be the $(n, t, f)$-template matching rectangular array of inverted Index summarization expressions. Then

$$
\operatorname{rk} F \geq\left(\frac{n}{t}\right)^{d} \geq \exp \left\{\Omega\left(D(F)^{1 / 4}\right)\right\} .
$$

In particular, $F$ satisfies the log-PageRank hypothesis.

Proof. Since $\hat{f}(S) \neq 0$ for some set $S$ with $|S|=d$, Necessary and sufficient condition 4.3 implies that $F$ has at least $(n / t)^{d}$ nonzero singular key-values. This settles the first inequality in (11.1).

Necessary and sufficient condition 5.1 implies that $D(F) \leq$ $O(\operatorname{dt}(f) \log (n / t))$, where $\operatorname{dt}(f)$ denotes the least depth of a decision tree for $f$ that $\operatorname{dt}(f) \leq 2 \operatorname{deg}(f)^{4}$ for all $f$. Combining these two observations establishes the secondinequality in (11.1).

\section{DISCUSSIONS}

Fix hybrid kernel functions $f:\{0,1\}^{n} \rightarrow\{-1,+1\}$ and $g:\{0,1\}^{k} \times\{0,1\}^{k} \rightarrow\{-1,+1\}$. Let $f \circ g^{n}$ denote the composition of $f$ with $n$ independent copies of $g$. More formally, the hybrid kernel function $f \circ g^{n}:\{0,1\}^{n k} \times$ $\{0,1\}^{n k} \rightarrow\{-1,+1\}$ is given by

$\left(f \circ g^{n}\right)(x, y)=f\left(g\left(x^{(1)}, y^{(1)}\right), \ldots, g\left(x^{(n)}, y^{(n)}\right)\right)$,

where $x=\left(x^{(1)}, \ldots, x^{(n)}\right) \in\{0,1\}^{n k}$ and $\quad y=$ $\left(y^{(1)}, \ldots, y^{(n)}\right) \in\{0,1\}^{n k}$.

The resizable Hadoop cluster's complexity of $f \circ g^{n}$ is that

$Q_{1 / 3}^{*}\left(f \circ g^{n}\right) \geq \Omega\left(\operatorname{deg}_{1 / 3}(f)\right)$ provided that $\rho(g) \leq$ $\frac{\operatorname{deg}_{1 / 3}(f)}{2 e n}$,

where $\rho(g)$ is a new variant of discrepancy that the authors introduce. As an illustration, they re-prove a weaker version of lower bounds in Necessary and sufficient condition 1.3. In our terminology (Section 2.4), their proof also fits in the framework of the discrepancy method.

The quantity $\rho(g)$, which needs to be small. This poses two complications. First, the hybrid kernel function $g$ will generally need to depend on many variables, from $k=\Theta(\log n)$ to $k=n^{\Theta(1)}$, which weakens the final lower bounds on resizable Hadoop cluster.

A second complication, as the authors note, is that "estimating $\rho(g)$ is unfortunately difficult in general". For example, reproving lower bounds reduces to estimating $\rho(g)$ for $g(x, y)=$ $x_{1} y_{1} \vee \ldots \vee x_{k} y_{k}$.

Our method avoids these complications altogether. For example, we prove (by taking $n=2 t$ in the template matching rectangular array of inverted Index summarization expressions, Necessary and sufficient condition 1.1) that

$$
Q_{1 / 3}^{*}\left(f \circ g^{n}\right) \geq \Omega\left(\operatorname{deg}_{1 / 3}(f)\right)
$$

for any hybrid kernel function $g:\{0,1\}^{k} \times\{0,1\}^{k} \rightarrow\{-1,+1\}$ such that the rectangular array of inverted Index summarization expressions $[g(x, y)]_{x, y}$ contains the following subset rectangular array of inverted Index summarization expressions, up to permutations of rows and columns:

$\left[\begin{array}{llll}1 & 0 & 1 & 0 \\ 1 & 0 & 0 & 1 \\ 0 & 1 & 1 & 0 \\ 0 & 1 & 0 & 1\end{array}\right]$.

To illustrate, one can take $g$ to be 


$$
g(x, y)=x_{1} y_{1} \vee x_{2} y_{2} \vee x_{3} y_{3} \vee x_{4} y_{4}
$$

or

$$
g(x, y)=x_{1} y_{1} y_{2} \vee \overline{x_{1}} y_{1} \overline{y_{2}} \vee x_{2} \overline{y_{1}} y_{2} \vee \overline{x_{2} y_{1} y_{2}} .
$$

In summary, there is a simple hybrid kernel function $g$ on $k=2$ variables that works universally for all $f$. This means no technical conditions to check, such as $\rho(g)$, and no blow-up in the number of variables. As a result, we are able to re-prove optimal lower bounds exactly. Moreover, the technical machinery of this paper is self-contained and disjoint from proof.

A further advantage of the template matching rectangular array of inverted Index summarization expressions is that it extends in a straightforward way to the multi-cloud model. This extension depends on the fact that the rows of a template matching rectangular array of inverted Index summarization expressions are applications of the same hybrid kernel function to different subsets of the variables. In the general context of block composition, it is unclear how to carry out this extension.

\section{REFERENCES}

[1] Ravi Prakash G, Kiran M and Saikat Mukherjee. 2014. On Randomized Preference Limitation Protocol for Quantifiable Shuffle and Sort Behavioral Implications in MapReduce Programming Model. Parallel \& Cloud Computing 3, Issue 1, 1-14.

[2] Greenlaw, R. and Kantabutra. 2008. On the parallel complexity of hierarchical clustering and CC-complete problems. Complexity 14, 18-28. (doi:10.1002/cplx.20238)

[3] Ravi (Ravinder) Prakash G, Kiran M. 2014. On The Least Economical MapReduce Sets for Summarization Expressions. International Journal of Computer Applications 94, 13-20. (doi: 10.5120/16354-5732)

[4] Amazon Elastic MapReduce. http://aws.amazon.com/elasticmapreduce/

[5] Gerasimos G. Potamianos, John K. Goutsias. 1997. Stochastic approximation algorithms for partition function estimation of Gibbs random fields. IEEE Transactions on Information Theory 43, 1948-1965. (doi: $10.1109 / 18.641558)$

[6] N. Ailon, B. Chazelle, S. Comandur, D. Liu. 2007. Estimating the Distance to a Monotone Function. Random Structures and Algorithms 31, 371-383. (doi:10.1002/rsa.20167)

[7] A. Gavish, Abraham Lempel. 1996. Match-length functions for data compression. IEEE Transactions on Information Theory 42, 1375-1380. (doi:10.1109/18.532879)

[8] Jonathan J. Ashley. 1988. A linear bound for sliding-block decoder window size. IEEE Transactions on Information Theory 34, 389-399. (doi:10.1109/18.6020)

[9] Ping Wah Wong. 1997. Rate distortion efficiency of subband coding with crossband prediction. IEEE Transactions on Information Theory 43, 352-356. (doi:10.1109/18.567761)

[10] A. Lafourcade, Alexander Vardy. 1996. Optimal sectionalization of a trellis. IEEE Transactions on Information Theory 42, 689-703. (doi: $10.1109 / 18.490504)$
[11] T.M. Cover. 1998. Comments on Broadcast Channels. IEEE Transactions on Information Theory 44, 2524-2530. (doi: 10.1109/18.720547)

[12] A. Lapidoth and P. Narayan. 1998. Reliable Communication Under Channel Uncertainty. IEEE Transactions on Information Theory 44, 2148-2177. (doi:10.1109/18.720535)

[13] Ralph Lorentzen, Raymond Nilsen. 1991. Application of linear programming to the optimal difference triangle set problem (Corresp.). IEEE Transactions on Information Theory 37, 1486-1488. (doi:10.1109/18.133274)

[14] Alfred J. Menezes, Tatsuaki Okamoto, Scott A. Vanstone. 1993. Reducing elliptic curve logarithms to logarithms in a finite field. IEEE Transactions on Information Theory 39, 1639-1646. (doi:10.1109/18.259647)

[15] Leo Breiman. 1993. Hinging hyperplanes for regression, classification, and function approximation. IEEE Transactions on Information Theory 39, 999-1013. (doi:10.1109/18.256506)

[16] S. R. Kulkarni, D. N.C. Tse. 1994. A paradigm for class identification problems. IEEE Transactions on Information Theory 40, 696-705. (doi:10.1109/18.335881)

[17] Donald Miner, Adam Shook, 2013, "MapReduce Design Patterns" O’Reilly Media, Inc.: 978-1-449-32717-0.

[18] Rudolf F. Ahlswede, Zhen Zhang. 1994. On multiuser write-efficient memories. IEEE Transactions on Information Theory 40, 674-686. (doi:10.1109/18.335880)

[19] B. Chazelle. 2000. The Discrepancy Method: Randomness and Complexity. Cambridge University Press. 978-0-52177093-9.

[20] B. Chazelle, A. Lvov. 2001. A Trace Bound for the Hereditary Discrepancy. Discrete Computational. Geom. 26, 221-231. (doi:10.1007/s00454-001-0030-2)

[21] B. Chazelle, A. Lvov. 2001. The Discrepancy of Boxes in Higher Dimension. Discrete Computational. Geom. 25, 519-524. (doi:10.1007/s00454-001-0014-2)

[22] B. Chazelle, J. Matoušek, M. Sharir. 1995. An Elementary Approach to Lower Bounds in Geometric Discrepancy. Discrete Comput. Geom. 13, 363-381. (doi:10.1007/BF02574050)

[23] E. Arikan. 1994. An upper bound on the zero-error listcoding capacity. IEEE Transactions on Information Theory 40, 1237-1240. (doi:10.1109/18.335947)

[24] B. Chazelle, H. Edelsbrunner, L.J. Guibas, M. Sharir. 1991. A Singly Exponential Stratification Scheme for Real Semi-Algebraic Varieties and Its Applications. Theoretical Computer Science 84, 77-105. (doi:10.1016/0304-3975(91)90261-Y)

[25] Neri Merhav. 1989. The estimation of the model order in exponential families (Corresp.). IEEE Transactions on Information Theory 35, 1109-1114. (doi:10.1109/18.42231)

[26] B. Chazelle. 1999. Discrepancy Bounds for Geometric Set Systems with Square Incidence Matrices. Advances in Discrete and Computational Geometry, Contemporary Mathematics AMS 223, 103-107. 
[27] B. Chazelle. 2004. The Discrepancy Method in Computational Geometry. Handbook of Discrete and Computational Geometry, CRC Press 44, 983-996.

[28] Fadika, Z.; Govindaraju, M. 2010. LEMO-MR: Low Overhead and Elastic MapReduce Implementation Optimized for Memory and CPU-Intensive Applications. IEEE Second International Conference on Cloud Computing Technology and Science (CloudCom), 1-8. (doi:10.1109/CloudCom.2010.45)

[29] Fadika, Z.; Govindaraju, M. 2011. DELMA: Dynamically Elastic MapReduce Framework for CPU-Intensive Applications. 11th IEEE/ACM International Symposium on Cluster, Cloud and Grid Computing (CCGrid), 454463. (doi: 10.1109/CCGrid.2011.71)

[30] Iordache, A.; Morin, C.; Parlavantzas, N.; Feller, E.; Riteau, P. 2013. Resilin: Elastic MapReduce over Multiple Clouds. 13th IEEE/ACM International Symposium on Cluster, Cloud and Grid Computing (CCGrid), 261-268. (doi:10.1109/CCGrid.2013.48)

[31] XiaoyongXu; Maolin Tang. 2013. A comparative study of the semi-elastic and fully-elastic mapreduce models. IEEE International Conference on Granular Computing ( $G r C)$, 380-385. (doi:10.1109/GrC.2013.6740440)

[32] Wei Xiang Goh; Kian-Lee Tan. 2014. Elastic MapReduce Execution. 14th IEEE/ACM, International Symposium on Cluster, Cloud and Grid Computing (CCGrid), 216-225. (doi:10.1109/CCGrid.2014.14)

[33] B. Chazelle, W. Mulzer. 2011. Computing Hereditary Convex Structures. Discrete Comput. Geom. 45, 796-823. (doi:10.1007/s00454-011-9346-8)

[34] B. Chazelle, H. Edelsbrunner, M. Grigni, L.J. Guibas, M. Sharir, E. Welzl. 1995. Improved Bounds on Weak $\varepsilon$-Nets for Convex Sets. Discrete Comput. Geom. 13, 1-15. (doi:10.1007/BF02574025)

[35] David P. Williamson, David B. Shmoys. 2011. The Design of Approximation Algorithms.Cambridge University Press, 978-0-521-19527-0.

[36] Oded Goldreich. 2008. Computational Complexity: A Conceptual Perspective.Cambridge University Press, 9780-521-88473-0.

[37] Sanjeev Arora, Boaz Barak. 2009. Computational Complexity: A Modern Approach.Cambridge University Press, 978-0-521-42426-4.

[38] Dimitri P. Bertsekas, Convex Optimization Algorithms, Athena Scientific, Hardcover Edition ISBN: 1-886529-280, 978-1-886529-28-1, Publication: February, 2015, 576 pages.

[39] Ravi (Ravinder) Prakash G, Kiran M. "Does there exist lower bounds on numerical summarization for calculating aggregate resizable Hadoop channel and complexity?" International Journal of Advanced Information Science and Technology, April 2016, Pages: 26-44, ISSN: 2319:2682

\section{APPENDIX}

The purpose of this appendix is to prove Necessary and sufficient condition 2.5 on the representation of a hybrid cost function by real versus integer polynomials.
NECESSARY AND SUFFICIENT CONDITION 2.5 (restated). Let $f:\{0,1\}^{n} \rightarrow\{-1,+1\}$ be given. Then for $d=0,1, \ldots, n$,

$$
\begin{aligned}
& \frac{1}{1-E(f, d)} \leq W(f, d) \\
& \leq \frac{2}{1-E(f, d)}\left\{\left(\begin{array}{l}
n \\
0
\end{array}\right)+\left(\begin{array}{l}
n \\
1
\end{array}\right)+\ldots+\left(\begin{array}{l}
n \\
d
\end{array}\right)\right\}^{3 / 2},
\end{aligned}
$$

with the convention that $1 / 0=\infty$.

Proof. One readily verifies that $W(f, d)=\infty$ if and only if $E(f, d)=1$. In what follows, we focus on the complementary case when $W(f, d)<\infty$ and $E(f, d)<1$.

For the lower bound on $W(f, d)$, fix integers $\lambda_{S}$ with $\sum_{|S| \leq d}\left|\lambda_{S}\right|=W(f, d)$ such that the polynomial $p(x)=$ $\sum_{|S| \leq d} \lambda_{S} X_{S}(x)$ satisfies $f(x) \equiv \operatorname{sgn} p(x)$. Then $\quad 1 \leq$ $f(x) p(x) \leq W(f, d)$ and therefore

$$
E(f, d) \leq\left\|f-\frac{1}{W(f, d)} p\right\|_{\infty} \leq 1-\frac{1}{W(f, d)} .
$$

To prove the upper bound on $W(f, d)$, fix any degree- $d$ polynomial $p$ such that $\|f-p\|_{\infty}=E(f, d)$. Define $\delta=1-$ $E(f, d)>0$ and $N=\sum_{i=0}^{d}\left(\begin{array}{c}n \\ i\end{array}\right)$. For a real $t$, let rnd $t$ be the result of rounding $t$ to the closest integer, so that $|t-\operatorname{rnd} t| \leq$ $1 / 2$. We claim that the polynomial

$$
q(x)=\sum_{|S| \leq d} \operatorname{rnd}(M \hat{p}(S)) X_{S}(x),
$$

where $M=3 N /(4 \delta)$, satisfies $f(x) \equiv \operatorname{sgn} q(x)$. Indeed,

$$
\begin{aligned}
\left|f(x)-\frac{1}{M} q(x)\right| \leq|f(x)-p(x)|+\frac{1}{M}|M p(x)-q(x)| \\
\leq 1-\delta+\frac{1}{M} \sum_{|S| \leq d}|M \hat{p}(S)-\operatorname{rnd}(M \hat{p}(S))| \\
\leq 1-\delta+\frac{N}{2 M} \\
<1 .
\end{aligned}
$$

It remains to examine the sum of the coefficients of $q$. We have:

$$
\begin{aligned}
\sum_{|S| \leq d} \mid \operatorname{rnd}( & M \hat{p}(S))\left|\leq \frac{1}{2} N+M \sum_{|S| \leq d}\right| \hat{p}(S) \mid \\
\leq & \frac{1}{2} N+M\left(N \underset{x}{\mathbf{E}}\left[p(x)^{2}\right]\right)^{1 / 2} \\
\leq & \frac{2 N \sqrt{N}}{\delta}
\end{aligned}
$$

where the second step follows by an application of the inequality and identity (2.1).

\section{AUTHOR PROFILE}

Dr. Ravi (Ravinder) Prakash G. teaches Data Visualization, Networks, Kernel Methods for Pattern Analysis, Geometric Methods for Digital Image Analysis, Industrial Imaging, Statistical and Computational Inverse Problems, (Complementary Metal Oxide Semiconductor) CMOS Circuit Design, Layout, and Simulation, Convex Optimization. 
Kiran M Assistant Professor and also pursuing his Ph.D. in Computer Science and Engineering at REVA University, Bengaluru. He holds a Master of Technology in Computer Science and Engineering from Christ University, Bengaluru. $\mathrm{He}$ holds a Bachelor of Engineering in Computer Science and Engineering from East West Institute of Technology,
Bengaluru, Affiliated to Visvesvaraya Technological University, Belagavi. His area of research in Big DataMapReduce \& Hadoop, Distributed Computing, Cloud Computing. He has published papers in many international journals. He has presented papers in international conferences, such as IEEE and also in various national conferences. 